DESY-08-036

2nd April 2008

\title{
Energy dependence of the charged multiplicity in deep inelastic scattering at HERA
}

\author{
ZEUS Collaboration
}

\begin{abstract}
The charged multiplicity distributions and the mean charged multiplicity have been investigated in inclusive neutral current deep inelastic ep scattering with the ZEUS detector at HERA, using an integrated luminosity of $38.6 \mathrm{pb}^{-1}$. The measurements were performed in the current region of the Breit frame, as well as in the current fragmentation region of the hadronic centre-of-mass frame. The KNO-scaling properties of the data were investigated and the energy dependence was studied using different energy scales. The data are compared to results obtained in $e^{+} e^{-}$collisions and to previous DIS measurements as well as to leading-logarithm parton-shower Monte Carlo predictions.
\end{abstract}




\section{The ZEUS Collaboration}

S. Chekanov, M. Derrick, S. Magill, B. Musgrave, D. Nicholass ${ }^{1}$, J. Repond, R. Yoshida Argonne National Laboratory, Argonne, Illinois 60439-4815, USA ${ }^{n}$

M.C.K. Mattingly

Andrews University, Berrien Springs, Michigan 49104-0380, USA

M. Jechow, N. Pavel ${ }^{\dagger}$

Institut für Physik der Humboldt-Universität zu Berlin, Berlin, Germany ${ }^{b}$

P. Antonioli, G. Bari, L. Bellagamba, D. Boscherini, A. Bruni, G. Bruni, F. Cindolo, M. Corradi, G. Iacobucci, A. Margotti, R. Nania, A. Polini

INFN Bologna, Bologna, Italy ${ }^{e}$

S. Antonelli, M. Basile, M. Bindi, L. Cifarelli, A. Contin, S. De Pasquale ${ }^{2}$, G. Sartorelli, A. Zichichi

University and INFN Bologna, Bologna, Italy ${ }^{e}$

D. Bartsch, I. Brock, H. Hartmann, E. Hilger, H.-P. Jakob, M. Jüngst, A.E. Nuncio-Quiroz, E. Paul ${ }^{3}$, U. Samson, V. Schönberg, R. Shehzadi, M. Wlasenko

Physikalisches Institut der Universität Bonn, Bonn, Germany ${ }^{b}$

N.H. Brook, G.P. Heath, J.D. Morris, A. Solomin

H.H. Wills Physics Laboratory, University of Bristol, Bristol, United Kingdom ${ }^{m}$

M. Capua, S. Fazio, A. Mastroberardino, M. Schioppa, G. Susinno, E. Tassi

Calabria University, Physics Department and INFN, Cosenza, Italy ${ }^{e}$

J.Y. Kim

Chonnam National University, Kwangju, South Korea

Z.A. Ibrahim, B. Kamaluddin, W.A.T. Wan Abdullah

Jabatan Fizik, Universiti Malaya, 50603 Kuala Lumpur, Malaysia ${ }^{r}$

Y. Ning, Z. Ren, F. Sciulli

Nevis Laboratories, Columbia University, Irvington on Hudson, New York 10027

J. Chwastowski, A. Eskreys, J. Figiel, A. Galas, M. Gil, K. Olkiewicz, P. Stopa, L. Zawiejski

The Henryk Niewodniczanski Institute of Nuclear Physics, Polish Academy of Sciences, Cracow, Poland ${ }^{i}$

L. Adamczyk, T. Bołd, I. Grabowska-Bołd, D. Kisielewska, J. Łukasik, M. Przybycień,

L. Suszycki

Faculty of Physics and Applied Computer Science, AGH-University of Science and Technology, Cracow, Poland ${ }^{p}$ 
A. Kotański ${ }^{4}$, W. Słomiński ${ }^{5}$

Department of Physics, Jagellonian University, Cracow, Poland

U. Behrens, C. Blohm, A. Bonato, K. Borras, R. Ciesielski, N. Coppola, S. Fang, J. Fourletova ${ }^{6}$,

A. Geiser, P. Göttlicher ${ }^{7}$, J. Grebenyuk, I. Gregor, T. Haas, W. Hain, A. Hüttmann,

F. Januschek, B. Kahle, I.I. Katkov, U. Klein ${ }^{8}$, U. Kötz ${ }^{3}$, H. Kowalski, E. Lobodzinska,

B. Löhr ${ }^{3}$, R. Mankel, I.-A. Melzer-Pellmann, S. Miglioranzi, A. Montanari, T. Namsoo,

D. Notz ${ }^{9}$, A. Parenti, L. Rinaldi ${ }^{10}$, P. Roloff, I. Rubinsky, R. Santamarta ${ }^{11}$, U. Schneekloth,

A. Spiridonov ${ }^{12}$, D. Szuba ${ }^{13}$, J. Szuba ${ }^{14}$, T. Theedt, G. Wolf ${ }^{3}$, K. Wrona, A.G. Yagües Molina,

C. Youngman, W. Zeuner ${ }^{9}$

Deutsches Elektronen-Synchrotron DESY, Hamburg, Germany

V. Drugakov, W. Lohmann, S. Schlenstedt

Deutsches Elektronen-Synchrotron DESY, Zeuthen, Germany

G. Barbagli, E. Gallo

INFN Florence, Florence, Italy ${ }^{e}$

P. G. Pelfer

University and INFN Florence, Florence, Italy ${ }^{e}$

A. Bamberger, D. Dobur, F. Karstens, N.N. Vlasov ${ }^{15}$

Fakultät für Physik der Universität Freiburg i.Br., Freiburg i.Br., Germany ${ }^{b}$

P.J. Bussey ${ }^{16}$, A.T. Doyle, W. Dunne, M. Forrest, M. Rosin, D.H. Saxon, I.O. Skillicorn

Department of Physics and Astronomy, University of Glasgow, Glasgow, United Kingdom ${ }^{m}$

I. Gialas ${ }^{17}$, K. Papageorgiu

Department of Engineering in Management and Finance, Univ. of Aegean, Greece

U. Holm, R. Klanner, E. Lohrmann, P. Schleper, T. Schörner-Sadenius, J. Sztuk, H. Stadie,

M. Turcato

Hamburg University, Institute of Exp. Physics, Hamburg, Germany ${ }^{b}$

C. Foudas, C. Fry, K.R. Long, A.D. Tapper

Imperial College London, High Energy Nuclear Physics Group, London, United Kingdom ${ }^{m}$

T. Matsumoto, K. Nagano, K. Tokushuku ${ }^{18}$, S. Yamada, Y. Yamazaki ${ }^{19}$

Institute of Particle and Nuclear Studies, KEK, Tsukuba, Japan ${ }^{f}$

A.N. Barakbaev, E.G. Boos ${ }^{3}$, N.S. Pokrovskiy, B.O. Zhautykov

Institute of Physics and Technology of Ministry of Education and Science of Kazakhstan, Almaty, Kazakhstan 
V. Aushev ${ }^{20}$, M. Borodin, I. Kadenko, A. Kozulia, V. Libov, M. Lisovyi, D. Lontkovskyi, I. Makarenko, Iu. Sorokin, A. Verbytskyi, O. Volynets

Institute for Nuclear Research, National Academy of Sciences, Kiev and Kiev National University, Kiev, Ukraine

D. Son

Kyungpook National University, Center for High Energy Physics, Daegu, South Korea ${ }^{g}$

J. de Favereau, K. Piotrzkowski

Institut de Physique Nucléaire, Université Catholique de Louvain, Louvain-la-Neuve, Belgium ${ }^{q}$

F. Barreiro, C. Glasman, M. Jimenez, L. Labarga, J. del Peso, E. Ron, M. Soares,

J. Terrón, M. Zambrana

Departamento de Física Teórica, Universidad Autónoma de Madrid, Madrid, Spain ${ }^{l}$

F. Corriveau, C. Liu, J. Schwartz, R. Walsh, C. Zhou

Department of Physics, McGill University, Montréal, Québec, Canada H3A 2T8 ${ }^{a}$

T. Tsurugai

Meiji Gakuin University, Faculty of General Education, Yokohama, Japan ${ }^{f}$

A. Antonov, B.A. Dolgoshein, D. Gladkov, V. Sosnovtsev, A. Stifutkin, S. Suchkov

Moscow Engineering Physics Institute, Moscow, Russia ${ }^{j}$

R.K. Dementiev, P.F. Ermolov, L.K. Gladilin, Yu.A. Golubkov, L.A. Khein, I.A. Korzhavina, V.A. Kuzmin, B.B. Levchenko ${ }^{21}$, O.Yu. Lukina, A.S. Proskuryakov, L.M. Shcheglova, D.S. Zotkin

Moscow State University, Institute of Nuclear Physics, Moscow, Russia ${ }^{k}$

I. Abt, A. Caldwell, D. Kollar, B. Reisert, W.B. Schmidke

Max-Planck-Institut für Physik, München, Germany

G. Grigorescu, A. Keramidas, E. Koffeman, P. Kooijman, A. Pellegrino, H. Tiecke, M. Vázquez ${ }^{9}$, L. Wiggers

NIKHEF and University of Amsterdam, Amsterdam, Netherlands ${ }^{h}$

N. Brümmer, B. Bylsma, L.S. Durkin, A. Lee, T.Y. Ling

Physics Department, Ohio State University, Columbus, Ohio 43210 ${ }^{n}$

P.D. Allfrey, M.A. Bell, A.M. Cooper-Sarkar, R.C.E. Devenish, J. Ferrando, B. Foster,

K. Korcsak-Gorzo, K. Oliver, S. Patel, V. Roberfroid ${ }^{22}$, A. Robertson, P.B. Straub,

C. Uribe-Estrada, R. Walczak

Department of Physics, University of Oxford, Oxford United Kingdom ${ }^{m}$

A. Bertolin, F. Dal Corso, S. Dusini, A. Longhin, L. Stanco

INFN Padova, Padova, Italy ${ }^{e}$ 
P. Bellan, R. Brugnera, R. Carlin, A. Garfagnini, S. Limentani

Dipartimento di Fisica dell' Università and INFN, Padova, Italy ${ }^{e}$

B.Y. Oh, A. Raval, J. Ukleja ${ }^{23}$, J.J. Whitmore ${ }^{24}$

Department of Physics, Pennsylvania State University, University Park, Pennsylvania $16802^{\circ}$

Y. Iga

Polytechnic University, Sagamihara, Japan ${ }^{f}$

G. D'Agostini, G. Marini, A. Nigro

Dipartimento di Fisica, Università 'La Sapienza' and INFN, Rome, Italy ${ }^{e}$

J.E. Cole, J.C. Hart

Rutherford Appleton Laboratory, Chilton, Didcot, Oxon, United Kingdom ${ }^{m}$

H. Abramowicz ${ }^{25}$, R. Ingbir, S. Kananov, A. Levy, A. Stern

Raymond and Beverly Sackler Faculty of Exact Sciences, School of Physics, Tel-Aviv

University, Tel-Aviv, Israel ${ }^{d}$

M. Kuze, J. Maeda

Department of Physics, Tokyo Institute of Technology, Tokyo, Japan ${ }^{f}$

R. Hori, S. Kagawa ${ }^{26}$, N. Okazaki, S. Shimizu, T. Tawara

Department of Physics, University of Tokyo, Tokyo, Japan ${ }^{f}$

R. Hamatsu, H. Kaji ${ }^{27}$, S. Kitamura ${ }^{28}$, O. Ota, Y.D. Ri

Tokyo Metropolitan University, Department of Physics, Tokyo, Japan ${ }^{f}$

M. Costa, M.I. Ferrero, V. Monaco, R. Sacchi, A. Solano

Università di Torino and INFN, Torino, Italy ${ }^{e}$

M. Arneodo, M. Ruspa

Università del Piemonte Orientale, Novara, and INFN, Torino, Italy ${ }^{e}$

S. Fourletov ${ }^{6}$, J.F. Martin, T.P. Stewart

Department of Physics, University of Toronto, Toronto, Ontario, Canada M5S 1A7 a

S.K. Boutle ${ }^{17}$, J.M. Butterworth, C. Gwenlan ${ }^{29}$, T.W. Jones, J.H. Loizides, M. Wing ${ }^{30}$

Physics and Astronomy Department, University College London, London, United Kingdom ${ }^{m}$

B. Brzozowska, J. Ciborowski ${ }^{31}$, G. Grzelak, P. Kulinski, P. Łużniak ${ }^{32}$, J. Malka ${ }^{32}$, R.J. Nowak,

J.M. Pawlak, T. Tymieniecka, A. Ukleja, A.F. Żarnecki

Warsaw University, Institute of Experimental Physics, Warsaw, Poland

M. Adamus, P. Plucinski ${ }^{33}$

Institute for Nuclear Studies, Warsaw, Poland 
Y. Eisenberg, D. Hochman, U. Karshon

Department of Particle Physics, Weizmann Institute, Rehovot, Israel ${ }^{c}$

E. Brownson, T. Danielson, A. Everett, D. Kçira, D.D. Reeder ${ }^{3}$, P. Ryan, A.A. Savin, W.H. Smith, H. Wolfe

Department of Physics, University of Wisconsin, Madison, Wisconsin 53706, USA ${ }^{n}$

S. Bhadra, C.D. Catterall, Y. Cui, G. Hartner, S. Menary, U. Noor, J. Standage, J. Whyte Department of Physics, York University, Ontario, Canada M3J 1Pз ${ }^{a}$ 
${ }^{1}$ also affiliated with University College London, UK

${ }^{2}$ now at University of Salerno, Italy

${ }^{3}$ retired

${ }^{4}$ supported by the research grant no. 1 P03B 04529 (2005-2008)

5 This work was supported in part by the Marie Curie Actions Transfer of Knowledge project COCOS (contract MTKD-CT-2004-517186)

${ }^{6}$ now at University of Bonn, Germany

${ }^{7}$ now at DESY group FEB, Hamburg, Germany

8 now at University of Liverpool, UK

${ }^{9}$ now at CERN, Geneva, Switzerland

10 now at Bologna University, Bologna, Italy

11 now at BayesForecast, Madrid, Spain

12 also at Institut of Theoretical and Experimental Physics, Moscow, Russia

13 also at INP, Cracow, Poland

14 also at FPACS, AGH-UST, Cracow, Poland

15 partly supported by Moscow State University, Russia

${ }^{16}$ Royal Society of Edinburgh, Scottish Executive Support Research Fellow

17 also affiliated with DESY, Germany

18 also at University of Tokyo, Japan

19 now at Kobe University, Japan

20 supported by DESY, Germany

${ }^{21}$ partly supported by Russian Foundation for Basic Research grant no. 05-02-39028NSFC-a

${ }^{22}$ EU Marie Curie Fellow

23 partially supported by Warsaw University, Poland

24 This material was based on work supported by the National Science Foundation, while working at the Foundation.

25 also at Max Planck Institute, Munich, Germany, Alexander von Humboldt Research Award

${ }^{26}$ now at KEK, Tsukuba, Japan

27 now at Nagoya University, Japan

28 Department of Radiological Science, Tokyo Metropolitan University, Japan

29 PPARC Advanced fellow

30 also at Hamburg University, Inst. of Exp. Physics, Alexander von Humboldt Research Award and partially supported by DESY, Hamburg, Germany

31 also at Łódź University, Poland

32 Łódź University, Poland

33 now at Lund Universtiy, Lund, Sweden

$\dagger$ deceased 
a supported by the Natural Sciences and Engineering Research Council of Canada (NSERC)

$b$ supported by the German Federal Ministry for Education and Research (BMBF), under contract numbers 05 HZ6PDA, 05 HZ6GUA, 05 HZ6VFA and 05 HZ4KHA

$c$ supported in part by the MINERVA Gesellschaft für Forschung GmbH, the Israel Science Foundation (grant no. 293/02-11.2) and the U.S.-Israel Binational Science Foundation

$d \quad$ supported by the German-Israeli Foundation and the Israel Science Foundation

$e$ supported by the Italian National Institute for Nuclear Physics (INFN)

$f$ supported by the Japanese Ministry of Education, Culture, Sports, Science and Technology (MEXT) and its grants for Scientific Research

$g$ supported by the Korean Ministry of Education and Korea Science and Engineering Foundation

$h \quad$ supported by the Netherlands Foundation for Research on Matter (FOM)

$i$ supported by the Polish State Committee for Scientific Research, project no. DESY/256/2006 - 154/DES/2006/03

$j$ partially supported by the German Federal Ministry for Education and Research $(\mathrm{BMBF})$

$k$ supported by RF Presidential grant N 8122.2006.2 for the leading scientific schools and by the Russian Ministry of Education and Science through its grant for Scientific Research on High Energy Physics

$l$ supported by the Spanish Ministry of Education and Science through funds provided by CICYT

$m$ supported by the Science and Technology Facilities Council, UK

$n$ supported by the US Department of Energy

$o$ supported by the US National Science Foundation. Any opinion, findings and conclusions or recommendations expressed in this material are those of the authors and do not necessarily reflect the views of the National Science Foundation.

$p$ supported by the Polish Ministry of Science and Higher Education as a scientific project (2006-2008)

$q$ supported by FNRS and its associated funds (IISN and FRIA) and by an Inter-University Attraction Poles Programme subsidised by the Belgian Federal Science Policy Office

$r$ supported by the Malaysian Ministry of Science, Technology and Innovation/Akademi Sains Malaysia grant SAGA 66-02-03-0048 


\section{Introduction}

The production of multi-hadronic final states in high-energy two-body collisions has long been a subject of great interest from experimental and theoretical points of view. The charged multiplicity at HERA has been measured previously by the H1 [1-4] and ZEUS [57] experiments. In this paper, new measurements by the ZEUS collaboration of the charged multiplicity in deep inelastic scattering (DIS) are presented.

Measurements are performed in the hadronic centre-of-mass (HCM) frame and the results are compared with those obtained in $e^{+} e^{-}$collisions, as well as with those from previous DIS experiments $[1,8-10]$. For the ep final state, differences are expected in the photon (current) and proton (target) fragmentation regions, due to the asymmetric nature of the reaction. The detector acceptance only allows the current fragmentation region to be measured.

Measurements of the charged multiplicity are also performed in the current region of the Breit frame, which should behave similarly to one hemisphere in $e^{+} e^{-}$collisions. Previous DIS results $[2,5,11]$ using $Q$, the virtuality of the exchanged photon, as the scale showed a reasonable agreement with $e^{+} e^{-}$data. However, this agreement degraded at values of $Q$ below $6-8 \mathrm{GeV}$. In this paper, the energy of the current region of the Breit frame is used as the scale to compare with $e^{+} e^{-}$data.

An alternative energy scale, the invariant mass of the hadronic system, has also been used in both the Breit and HCM frames. The results using this variable are also compared to results from $e^{+} e^{-}$collisions.

\section{Experimental set-up}

The data were collected with the ZEUS detector during the 1996 and 1997 running periods, when HERA operated with protons of energy $E_{p}=820 \mathrm{GeV}$ and positrons of energy $E_{e}=27.5 \mathrm{GeV}$, and correspond to an integrated luminosity of $38.6 \pm 0.6 \mathrm{pb}^{-1}$.

The ZEUS detector is described in detail elsewhere [12]. The most important components used in the current analysis were the central tracking detector and the calorimeter.

Charged particles were tracked in the central tracking detector (CTD) [13], which operated in a magnetic field of $1.43 \mathrm{~T}$ provided by a thin superconducting coil. The CTD consisted of 72 cylindrical drift chamber layers, organised in 9 superlayers covering the polar-angle ${ }^{1}$

\footnotetext{
${ }^{1}$ The ZEUS coordinate system is a right-handed Cartesian system, with the $Z$ axis pointing in the proton beam direction, referred to as the "forward direction", and the $X$ axis pointing left towards the centre of HERA. The coordinate origin is at the nominal interaction point.
} 
region $15^{\circ}<\theta<164^{\circ}$. The transverse-momentum resolution for full-length tracks was $\sigma\left(p_{T}\right) / p_{T}=0.0058 p_{T} \oplus 0.0065 \oplus 0.0014 / p_{T}$, with $p_{T}$ in $\mathrm{GeV}$.

The high-resolution uranium-scintillator calorimeter (CAL) [14] consisted of three parts: the forward (FCAL), the barrel (BCAL) and the rear (RCAL) calorimeters. Each part was subdivided transversely into towers and longitudinally into one electromagnetic section (EMC) and either one (in RCAL) or two (in BCAL and FCAL) hadronic sections (HAC). The smallest subdivision of the calorimeter was called a cell. The CAL energy resolutions, as measured under test-beam conditions, were $\sigma(E) / E=0.18 / \sqrt{E}$ for electrons and $\sigma(E) / E=0.35 / \sqrt{E}$ for hadrons, with $E$ in $\mathrm{GeV}$.

\section{Data selection}

Deep inelastic scattering events were selected by requiring that the outgoing positron was measured in the CAL. The scattered-positron identification was based on a neural-network algorithm using the CAL information [15].

For the reconstruction of the photon virtuality, $Q^{2}$, Bjorken $x$, and the $\gamma^{*} P$ centre-of-mass energy, $W$, the double angle (DA) method was chosen, in which the scattered-positron angle, $\theta_{e}$, and the hadronic angle $\gamma_{H}$ are used [16]. In the naive quark-parton model, $\gamma_{H}$ is the angle of the scattered massless quark in the laboratory frame.

For each event, the measurement of the charged multiplicity was performed using tracks reconstructed in the CTD. The energy of the hadronic final state was measured using a combination of track and CAL information, excluding the cells and the track associated with the scattered positron. The selected tracks and CAL clusters were treated as massless Energy Flow Objects (EFOs) [17]. The clustering of objects was done according to the Snowmass convention [18]. The transverse momentum, $p_{T}$, of each EFO was required to be greater than $0.15 \mathrm{GeV}$.

The event selection criteria were:

- $E_{e^{\prime}}>12 \mathrm{GeV}$, where $E_{e^{\prime}}$ is the energy of the scattered positron, to select neutral current DIS events;

- $y_{e} \leq 0.95$, where $y_{e}$ is the scaling variable $y$ as determined from the energy and polar angle of the scattered positron. This cut reduces the photoproduction background;

- $y_{\mathrm{JB}} \geq 0.04$, where $y_{\mathrm{JB}}$ is the estimate of $y$ using the Jacquet-Blondel (JB) method [19]. It is defined as $y_{\mathrm{JB}}=\sum_{h}\left(E_{h}-P_{Z_{h}}\right) / 2 E_{e}$, where the sum runs over all EFOs and $E_{h}$ and $P_{Z_{h}}$ are the energies and longitudinal momenta of the EFOs respectively. This requirement guarantees sufficient accuracy for the DA reconstruction method; 
- $35 \leq \delta \leq 60 \mathrm{GeV}$, where $\delta=\sum_{i}\left(E_{i}-P_{Z_{i}}\right)$ and the sum runs over all EFOs and the scattered positron, to remove photoproduction events and events with large radiative corrections;

- $R \geq 25 \mathrm{~cm}$, where $R$ is the distance from the beam axis to the impact position of the scattered positron on the face of the CAL. This ensured that the positron was fully contained within the detector and its position reconstructed with sufficient accuracy;

- $\left|Z_{\mathrm{vtx}}\right|<50 \mathrm{~cm}$, where $Z_{\mathrm{vtx}}$ is the longitudinal position of the vertex, to reduce background events from non-ep collisions.

To ensure high-quality tracks reconstructed with high efficiency, the following requirements were made:

- the tracks had to pass through at least three CTD superlayers;

- the tracks had to be associated with the primary event vertex;

- the tracks were restricted to the region $\left|\eta_{\mathrm{LAB}}\right| \leq 1.75$, where $\eta_{\mathrm{LAB}}$ is the pseudorapidity of the tracks in the laboratory frame;

- the tracks had $p_{T}>0.15 \mathrm{GeV}$.

The analysis was restricted to the kinematic range $Q^{2}>25 \mathrm{GeV}^{2}$ and $70<W<225 \mathrm{GeV}$.

\section{Analysis method}

Due to the large asymmetry of the beam energies at HERA, a large fraction of the hadronic final state close to the proton direction lies outside the detector acceptance. Therefore only hadrons belonging to the current fragmentation regions of the HCM and Breit frames were used in this analysis. The boost to the corresponding reference frames was calculated using the positron four-momentum taken from the DA method.

\subsection{Breit frame}

In the Breit frame, which is defined by the condition that the momentum of the exchanged virtual boson is purely spacelike, $\boldsymbol{q}=(0,0,-Q)$, the particles produced in the interaction can be assigned to one of two regions: the current region if their longitudinal momentum in the Breit frame is negative, and the target region if their longitudinal momentum is positive. The hadronic system of the current region used in this analysis is almost fully (about 95\%) contained within the acceptance of the CTD. 
Previous analyses compared the mean charged multiplicity $\left\langle n_{\mathrm{ch}}\right\rangle$ as a function of $Q$ to $\left\langle n_{\mathrm{ch}}\right\rangle / 2$ as a function of $\sqrt{s}$ in $e^{+} e^{-}$collisions [2,5]. For values of $Q>8 \mathrm{GeV}$, reasonable agreement was observed, while some disagreement was found for $Q<8 \mathrm{GeV}$. The difference can be understood in terms of higher-order processes [20], which change the available energy in the current region of the Breit frame, $E_{\mathrm{B}}^{\mathrm{cr}}$, which is no longer equal to $Q / 2$. In this analysis the quantity $2 \cdot E_{\mathrm{B}}^{\mathrm{cr}}$ is used as a scale. On an event-by-event basis this method should compensate for particles and their corresponding energies migrating between current and target regions of the Breit frame.

\subsection{Hadronic centre-of-mass frame}

In the HCM frame, the exchanged virtual boson has four-momentum $\boldsymbol{q}=\{E, p\}=$ $\left(\frac{W^{2}-Q^{2}}{2 W}, 0,0, \frac{W^{2}+Q^{2}}{2 W}\right)$. The hadronic final state is separated into the photon (current) and proton (target) fragmentation regions. About $60-80 \%$ of the current region of the HCM frame is contained within the acceptance of the CTD.

The multiplicity in the HCM frame in DIS is usually studied as a function of $W[1,8-10]$. At HERA, the energy in the current region of the HCM frame, $E_{\mathrm{HCM}}^{\mathrm{cr}}$, coincides with $W / 2$ within $0.3-0.4 \%$. Thus, for practical reasons, $W$ was used as the energy scale.

\subsection{Invariant mass of the hadronic system}

Charged multiplicities in the current region of both the Breit and HCM frames were also measured as functions of the invariant mass:

$$
M_{\mathrm{eff}}^{2}=\left(\sum_{i} E_{i}\right)^{2}-\left(\sum_{i} P_{X_{i}}\right)^{2}-\left(\sum_{i} P_{Y_{i}}\right)^{2}-\left(\sum_{i} P_{Z_{i}}\right)^{2}
$$

where the sum runs over all charged and neutral particles of the corresponding hadronic system.

\section{Monte Carlo models, acceptance corrections and systematic errors}

Samples of neutral current DIS events were generated using the colour-dipole model as implemented in ARIAdne 4.12 [21] or with the MEPS model of LePto 6.5 [22]. Both programs were interfaced to HeRACLES 4.6.1 [23] using the DJANGOH 1.1 [24] program. 
Both Ariadne and LePto use the Lund string model [25] for the hadronisation. The cluster hadronisation model as implemented in HERWIG 6.1 [26] was used to estimate the effect of different hadronisation schemes on the unfolding procedure. The minimum transverse momentum of outgoing partons in the hard interaction and of partons participating in multi-parton interactions is adjustable in HERWIG using the parameter $p_{T}^{\min }$. The parameter was tuned in this analysis to improve the description of the detector distributions. The best agreement was found for $p_{T}^{\min }=2.5 \mathrm{GeV}[27]$.

The corrections applied to the data accounted for the effects of acceptance and resolution of the detector, event selection cuts, QED-radiative effects, track reconstruction, track selection cuts, and energy losses in the inactive material in front of the calorimeter in the case of the energy measurement. Finally the multiplicity distributions were corrected using a matrix unfolding method as described in earlier studies [5].

The generated events were passed through a full simulation of the detector, using a program based on GEANT 3.13 [28], and processed and selected with the same programs as used for the data. The simulated samples were used to determine the response of the detector and to evaluate the correction factors necessary to obtain hadron-level quantities. The hadron level is defined by those hadrons with lifetime $\tau \geq 3 \cdot 10^{-10}$ seconds. In order to compare the results to different experiments, corrections were calculated both including and not including the decay products of $K_{S}^{0}$ and $\Lambda$.

The dominant systematic uncertainty in this analysis arises from the simulation of the hadronic final state. To correct the data, the average of the correction factors from the Ariadne and Herwig MC programs was used. One half of the difference, as large as $5 \%$, was assigned to the systematic uncertainties.

Other sources of uncertainty are (typical values of the uncertainties are shown in parentheses): event reconstruction and selection $(<0.5 \%)$, track reconstruction and selection $(<0.5 \%)$, and the uncertainty due to variation of the $Q^{2}$ cut by its resolution $(<1.7 \%)$. The uncertainty due to contamination from diffractive events is negligible. The individual systematic uncertainties were added in quadrature.

\section{Results}

\subsection{Multiplicity distributions}

The multiplicity distributions in the current region of the Breit frame are presented in Fig. 1 and Table 1 and in Fig. 2 and Table 2 in bins of $2 \cdot E_{\mathrm{B}}^{\mathrm{cr}}$ and in bins of $M_{\text {eff }}$ respectively. The kinematic range of the analysis restricts the $M_{\text {eff }}$ measurements in the current region of the Breit frame to a maximum value of about $20 \mathrm{GeV}$. The predictions of 
Ariadne, Lepto and Herwig are also shown. All three MC models generally describe the data, but ARIADNE gives the best description. In all Figures and Tables the chargedparticle decay products of $K_{S}^{0}$ and $\Lambda$ are included, unless otherwise stated.

For a given bin of energy, the multiplicities as a function of $2 \cdot E_{\mathrm{B}}^{\mathrm{cr}}$ and $M_{\mathrm{eff}}$ differ by approximately a factor of two. This is due to the fact that $2 \cdot E_{\mathrm{B}}^{\mathrm{cr}}$ (as well as $Q$ or $W$ ) characterise the total centre-of-mass energy of the system of which only one hemisphere is measured. On the other hand the $M_{\text {eff }}$ method measures the multiplicity of the system with respect to the corresponding invariant mass.

The multiplicity distributions in the current region of the HCM frame are presented in Fig. 3 and Table 3 in bins of $W$. Both ARIAdne and Lepto predict similar $W$ distributions and give a reasonable description of the data. HERWIG predicts longer tails for the multiplicity distributions in bins of $W$. This leads to higher average multiplicities, affecting the unfolded multiplicity values and increasing the systematic uncertainties of the measurement. The ARIADnE predictions vary slightly from both LEPTO and HERWiG at the rising edge of the distributions, which also leads to an increase of the systematic uncertainties.

The multiplicity distributions in the current region of the HCM frame in bins of $M_{\text {eff }}$ are presented in Fig. 4 and Table 4. To minimise the extrapolation both in multiplicity and $M_{\text {eff }}$, an additional requirement, $\left|\eta_{\mathrm{LAB}}\right|<1.75$, was applied at the hadron level. None of the MC models shown in Fig. 4 give a complete description of the data. This is most visible at higher values of $M_{\text {eff }}$.

\subsection{KNO scaling}

The multiplicity distributions are expected to scale with energy as discussed in detail elsewhere [29]. A commonly used form of the scaling, from KNO [30], is shown in Figs. 58 , where the product of the multiplicity distribution $P\left(n_{\mathrm{ch}}\right)$ with average multiplicity $\left\langle n_{\mathrm{ch}}\right\rangle,\left\langle n_{\mathrm{ch}}\right\rangle P\left(n_{\mathrm{ch}}\right)$, is shown as a function of $n_{\mathrm{ch}} /\left\langle n_{\mathrm{ch}}\right\rangle$.

In Fig. 5(a), the KNO distributions measured in bins of $W$ in the current region of the HCM frame are shown. Within the uncertainties, the distributions measured in three bins of $W$ agree. They also agree well with the average distribution, which was calculated using data for the entire $W$ region, $70<W<225 \mathrm{GeV}$. This average KNO spectrum, presented as a histogram, is shown in Figs. 5 and 6 as a reference KNO distribution.

The reference KNO distribution is compared to the measurements in the current region of the Breit frame in Figs. 5(b) and 5(c) in bins of $2 \cdot E_{\mathrm{B}}^{\mathrm{cr}}$. For values of $2 \cdot E_{\mathrm{B}}^{\mathrm{cr}}>12 \mathrm{GeV}$, as shown in Fig. $5(\mathrm{c})$, the measurements are in reasonable agreement with the reference KNO histogram. For lower values of $2 \cdot E_{\mathrm{B}}^{\mathrm{cr}}$, as demonstrated in Fig. $5(\mathrm{~b})$, the distributions do 
not follow the KNO-scaling behaviour; they have different shapes, but approach the KNO curve with increasing values of $2 \cdot E_{\mathrm{B}}^{\mathrm{cr}}$.

In Fig. 6, the KNO distributions measured in bins of $M_{\text {eff }}$ in the current region of the HCM frame are presented. The multiplicity distributions in bins of $M_{\text {eff }}$ do not follow the same KNO scaling observed for measurements as functions of $W$ or $2 \cdot E_{\mathrm{B}}^{\mathrm{cr}}$, but do demonstrate scaling behaviour for $M_{\text {eff }}$ values above $8 \mathrm{GeV}$. The measurements at $M_{\text {eff }}<4 \mathrm{GeV}$ in the current regions of both the Breit and HCM frames behave differently from the measurements at the higher values of $M_{\text {eff }}$.

Figure 7 shows a comparison of the KNO distributions from ZEUS with results obtained in $e^{+} e^{-}$collisions. The measurements in bins of $2 \cdot E_{\mathrm{B}}^{\mathrm{cr}}$, for $2 \cdot E_{\mathrm{B}}^{\mathrm{cr}}>12 \mathrm{GeV}$, and in bins of $W$ are plotted together and compared with measurements in one hemisphere of $e^{+} e^{-}$. In Fig. 7(a), a comparison with results from the TASSO collaboration [31] in the energy range $14<\sqrt{s_{e e}}<44 \mathrm{GeV}$ is shown. At LEP only DELPHI [32] and OPAL [33] performed measurements in a single hemisphere at $\sqrt{s_{e e}}=91.2 \mathrm{GeV}$. A comparison with the present data is shown in Fig. 7(b). The systematic uncertainties are not shown, but within statistical uncertainties there is a remarkable agreement between ep and $e^{+} e^{-}$ results. However, the LEP data differ somewhat from the present measurement in the peak region and at very low values of $n_{\mathrm{ch}} /\left\langle n_{\mathrm{ch}}\right\rangle$.

The data as a function of $M_{\text {eff }}$, for $M_{\text {eff }}>8 \mathrm{GeV}$, are compared with the $e^{+} e^{-}$measurements for the whole event in Fig. 8. Both the TASSO and LEP [32-34] data $(91.2<$ $\left.\sqrt{s_{e e}}<209 \mathrm{GeV}\right)$ agree with the present measurement.

\subsection{Mean charged multiplicity}

Figure 9 and Table 5 show the mean charged multiplicity, $\left\langle n_{\mathrm{ch}}\right\rangle$, in the current region of the HCM frame as a function of $W$ and the mean charged multiplicity in the current region of the Breit frame as a function of $2 \cdot E_{\mathrm{B}}^{\mathrm{cr}}$. The $K_{S}^{0}$ and $\Lambda$ hadrons were considered stable in Fig. 9, where the data are compared with results of previously published HERA measurements $[1,2,4,5]$. As expected, at low values of $2 \cdot E_{\mathrm{B}}^{\mathrm{cr}}$, the measurement differs with those as a function of $Q$ (see Section 4.1). At higher values of $2 \cdot E_{\mathrm{B}}^{\mathrm{cr}}$ the data agree within the experimental uncertainties with the previous ZEUS and H1 measurements, but lie systematically above them. The data are in good agreement with the ARIADNE and Lepto predictions. The Herwig predictions also describe the data but are below those from ARIADne and Lepto. In the current region of the HCM, the measurement agrees well, with improved statistical and systematic uncertainties, with the earlier H1 results. The ARIADne and LePto predictions agree with the data. HeRwig predicts a very different slope, with much higher multiplicities at higher energies; with increasing energy the agreement with data degrades. 
The mean charged multiplicities in the current regions of the Breit and HCM frames are presented in Fig. 10 and Table 6 as a function of the invariant mass of the corresponding hadronic system, $M_{\text {eff }}$. In Figs. $10(\mathrm{a})$ and $10(\mathrm{~b})$, the multiplicities are compared to the MC predictions. All three MC models describe the data reasonably well; however in the last $M_{\text {eff }}$ bin in the current region of the HCM, the HERWIG prediction is too high. In Fig. 10(c), both measurements are shown together and compared with different MC curves calculated using the ARIADNE MC. The measurements in the Breit and HCM frames agree at values of $M_{\text {eff }}$ less than $10 \mathrm{GeV}$. Above this value, $\left\langle n_{\mathrm{ch}}\right\rangle$ rises much faster with $M_{\text {eff }}$ in the current region of the HCM frame than in the current region of the Breit frame. Since the HCM measurement was restricted in $\eta$, a separate ARIADNE calculation was performed in the total current region of the HCM frame. The difference is small, although the rise of $\left\langle n_{\mathrm{ch}}\right\rangle$ with $M_{\text {eff }}$ is faster in the total current region of the HCM frame.

Figure $10(\mathrm{c})$ also shows $2 \cdot\left\langle n_{\mathrm{ch}}\right\rangle$ as a function of $2 \cdot E_{\mathrm{B}}^{\mathrm{cr}}$. This measurement exhibits the same behaviour as $\left\langle n_{\mathrm{ch}}\right\rangle$ as a function of $M_{\mathrm{eff}}$ in the current region of the Breit frame but differs from that in the HCM frame. The multiplicity in the current region of the HCM frame rises much faster with the invariant mass than with $W$.

Finally, Fig. 11 combines the mean charged multiplicities measured in the current regions of the Breit and HCM frames as functions of the respective energy scales, $2 \cdot E_{\mathrm{B}}^{\mathrm{cr}}$ and $W$. Also shown are the measurements from $e^{+} e^{-}[31-35]$ and fixed-target [8-10] experiments. The fixed-target data were scaled by a factor two, since they only measure one hemisphere and by a factor 1.08, to correct for the decays of the $K_{S}^{0}$ and $\Lambda$, as estimated using ARIADNE MC.

The measurements presented in this paper show good overall agreement with those from other experiments, exhibiting approximately the same dependence of the mean charged multiplicity on the respective energy scale. At low values of the energy, the measurement as a function of $2 \cdot E_{\mathrm{B}}^{\mathrm{cr}}$ agrees well with $e^{+} e^{-}$data, in contrast to the previous measurements as a function of $Q[2,5]$. The measurements in the current region of the HCM agree with the LEP data, but are systematically below them. The data from fixed-target DIS experiments [1,8-10] deviate from the observed energy dependence at energies above $15 \mathrm{GeV}$. The ARIadne MC prediction generally describes the energy dependence of the data over the entire region. However, the prediction in the HCM frame is generally lower than the data and than the prediction in the Breit frame. The Herwig MC model does not give a good overall description of the data. 


\section{$7 \quad$ Summary and conclusions}

The charged multiplicity distributions and the mean charged multiplicity have been investigated in inclusive neutral current deep inelastic ep scattering in the kinematic range $Q^{2}>25 \mathrm{GeV}^{2}$ and $70<W<225 \mathrm{GeV}$ in terms of different energy scales. The scale $2 \cdot E_{\mathrm{B}}^{\mathrm{cr}}$, was used in the current region of the Breit frame. In the current region of the HCM frame, $W$ was used and the invariant mass, $M_{\text {eff }}$, was used in both frames.

In terms of KNO scaling, the charged multiplicities in the current regions of the Breit and $\mathrm{HCM}$ frames exhibit the same behaviour as those in one hemisphere of $e^{+} e^{-}$collisions when $2 \cdot E_{\mathrm{B}}^{\mathrm{cr}}$ or $W$ are considered. When the energy scale $M_{\mathrm{eff}}$ is used, the charged multiplicities exhibit the same KNO-scaling behaviour as those for the whole $e^{+} e^{-}$event.

The mean charged multiplicity in the current region of the Breit frame scales with $M_{\text {eff }}$ in the same way as $2 \cdot\left\langle n_{\mathrm{ch}}\right\rangle$ scales with $2 \cdot E_{\mathrm{B}}^{\mathrm{cr}}$ and, therefore, as $\left\langle n_{\mathrm{ch}}\right\rangle$ scales with $\sqrt{s_{e e}}$ in $e^{+} e^{-}$collisions. The mean charged multiplicity in the current region of HCM frame as a function of $M_{\text {eff }}$ rises faster than that in the current region of the Breit frame.

The energy scale $2 \cdot E_{\mathrm{B}}^{\mathrm{cr}}$, rather than $Q$, gives better agreement between the mean charged multiplicity measured in the current region of the Breit frame and that measured in $e^{+} e^{-}$ collisions. The measurements of $\left\langle n_{\mathrm{ch}}\right\rangle$ as a function of $W$ agree, within the uncertainties, with the data from $e^{+} e^{-}$collisions. When using these scales, ep DIS data can be consistently compared with data from $e^{+} e^{-}, \mu P$ and $\nu P$ scattering over a wide energy region.

\section{Acknowledgements}

We are grateful to the DESY directorate for their strong support and encouragment. The effort of the HERA machine group is gratefully acknowledged. We thank the DESY computing and network services for their support. The design, construction and installation of the ZEUS detector has been made possible by the efforts of many people not listed as authors. 


\section{References}

[1] H1 Coll., S. Aid et al., Z. Phys. C 72, 573 (1996).

[2] H1 Coll., C. Adloff et al., Nucl. Phys. B 504, 3 (1997).

[3] H1 Coll., C. Adloff et al., Eur. Phys. J. C 5, 439 (1998).

[4] H1 Coll., F.D. Aaron et al., Phys. Lett. B 654, 148 (2007).

[5] ZEUS Coll., M. Derrick et al., Z. Phys. C 67, 93 (1995).

[6] ZEUS Coll., J. Breitweg et al., Eur. Phys. J. C 11, 251 (1999).

[7] ZEUS Coll., S. Chekanov et al., Phys. Lett. B 510, 36 (2001).

[8] E665 Coll., M.R. Adams et al., Z. Phys. C 76, 441 (1997).

[9] EMC Coll., M. Arneodo et al., Z. Phys. C 35, 335 (1987).

[10] WA21 Coll., G.T. Jones et al., Z. Phys. C 54, 45 (1992).

[11] NOMAD Coll., J. Altegoer et al., Phys. Lett. B 445, 439 (1999).

[12] ZEUS Coll., U. Holm (ed.), The ZEUS Detector. Status Report (unpublished), DESY (1993), available on http://www-zeus.desy.de/bluebook/bluebook.html.

[13] N. Harnew et al., Nucl. Inst. Meth. A 279, 290 (1989);

B. Foster et al., Nucl. Phys. Proc. Suppl. B 32, 181 (1993);

B. Foster et al., Nucl. Inst. Meth. A 338, 254 (1994).

[14] M. Derrick et al., Nucl. Inst. Meth. A 309, 77 (1991);

A. Andresen et al., Nucl. Inst. Meth. A 309, 101 (1991);

A. Caldwell et al., Nucl. Inst. Meth. A 321, 356 (1992);

A. Bernstein et al., Nucl. Inst. Meth. A 336, 23 (1993).

[15] H. Abramowicz, A. Caldwell and R. Sinkus, Nucl. Inst. Meth. A 365, 508 (1995).

[16] S. Bentvelsen, J. Engelen and P. Kooijman, Proc. Workshop on Physics at HERA, W. Buchmüller and G. Ingelman (eds.), Vol. 1, p. 23. Hamburg, Germany, DESY (1992).

[17] G.M. Briskin, Ph.D. Thesis, Tel Aviv University, 1998 (unpublished).

[18] J.E. Huth et al., Research Directions for the Decade. Proceedings of Summer Study on High Energy Physics, 1990, E.L. Berger (ed.), p. 134. World Scientific (1992). Also in preprint FERMILAB-CONF-90-249-E.

[19] F. Jacquet and A. Blondel, Proc. the Study of an eP Facility for Europe, U. Amaldi (ed.), p. 391 (1979). Also in preprint DESY 79-48.

[20] K.H. Streng, T.F. Walsh and P.M. Zerwas, Z. Phys. C 2, 237 (1979). 
[21] L. Lönnblad, Comp. Phys. Comm. 71, 15 (1992).

[22] G. Ingelman, A. Edin and J. Rathsman, Comp. Phys. Comm. 101, 108 (1997).

[23] A. Kwiatkowski, H. Spiesberger and H.-J. Möhring, Comp. Phys. Comm. 69, 155 (1992). Also in Proc. Workshop Physics at HERA, 1991, DESY, Hamburg; H. Spiesberger, An Event Generator for ep Interactions at HERA Including Radiative Processes (Version 4.6), 1996, available on

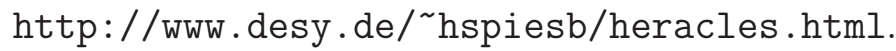

[24] H. Spiesberger, HeRACles and DJAngOH: Event Generation for ep Interactions at HERA Including Radiative Processes, 1998, available on http://www . desy.de/ ^hspiesb/djangoh.html;

K. Charchula, G.A. Schuler and H. Spiesberger, Comp. Phys. Comm. 81, 381 (1994).

[25] B. Andersson et al., Phys. Rep. 97, 31 (1983).

[26] G. Marchesini et al., Comp. Phys. Comm. 67, 465 (1992).

[27] ZEUS Coll., S. Chekanov et al., Eur. Phys. J. C 50, 283 (2007).

[28] R. Brun et al. (ed.), Geant3. Technical Report CERN-DD/EE/84-1, CERN (1987).

[29] M. Gazdzicki et al., Mod. Phys. Lett. A 6, 981 (1991).

[30] Z. Koba, H.B. Nielsen and P. Olesen, Nucl. Phys. B 40, 317 (1972).

[31] TASSO Coll., W. Braunschweig et al., Z. Phys. C 45, 193 (1989).

[32] DELPHI Coll., P. Abreu et al., Z. Phys. C 50, 185 (1991).

[33] OPAL Coll., P.D. Acton et al., Z. Phys. C 35, 539 (1991).

[34] ALEPH Coll., D. Decamp et al., Phys. Lett. B 273, 181 (1991);

ALEPH Coll., D. Buskulic et al., Z. Phys. C 73, 409 (1997);

DELPHI Coll., P. Abreu et al., Phys. Lett. B 372, 172 (1996);

DELPHI Coll., P. Abreu et al., Phys. Lett. B 416, 233 (1998);

DELPHI Coll., P. Abreu et al., Eur. Phys. J. C 18, 203 (2000);

L3 Coll., P. Achard et al., Phys. Rept. 399, 71 (2004);

L3 Coll., P. Achard et al., Phys. Lett. B 577, 109 (2003);

L3 Coll., M. Acciarri et al., Phys. Lett. B 371, 137 (1996);

L3 Coll., M. Acciarri et al., Phys. Lett. B 404, 390 (1997);

L3 Coll., M. Acciarri et al., Phys. Lett. B 444, 569 (1998);

OPAL Coll., G. Alexander et al., Z. Phys. C 72, 191 (1996);

OPAL Coll., K. Ackerstaff et al., Z. Phys. C 75, 193 (1997);

OPAL Coll., G. Abbiendi et al., Eur. Phys. J. C 16, 185 (1999). 
[35] MARK I Coll., V. Luth et al., Phys. Lett. B 70, 120 (1977);

JADE Coll., W. Bartel et al., Z. Phys. C 20, 187 (1983);

HRS Coll., M. Derrick et al., Phys. Rev. D 34, 3304 (1986). 


\begin{tabular}{|c|c|c|c|c|c|c|c|}
\hline $2 \cdot E_{\mathrm{B}}^{\mathrm{cr}}(\mathrm{GeV})$ & $1.5-4$ & $4-8$ & $8-12$ & $12-20$ & $20-30$ & $30-45$ & $45-100$ \\
\hline$n_{\mathrm{ch}}=0$ & $12.33 \pm 0.25_{-0.23}^{+0.21}$ & $4.34 \pm 0.09_{-0.65}^{+0.65}$ & $1.83 \pm 0.10_{-0.56}^{+0.57}$ & $0.81 \pm 0.03_{-0.26}^{+0.26}$ & $0.33 \pm 0.03_{-0.10}^{+0.10}$ & $0.23 \pm 0.05_{-0.09}^{+0.09}$ & $0.17 \pm 0.09_{-0.09}^{+0.09}$ \\
\hline 1 & $35.79 \pm 0.46_{-0.35}^{+0.18}$ & $17.44 \pm 0.19_{-0.72}^{+0.71}$ & $8.29 \pm 0.20_{-0.77}^{+0.78}$ & $4.35 \pm 0.06_{-0.44}^{+0.44}$ & $2.23 \pm 0.08_{-0.33}^{+0.33}$ & $1.45 \pm 0.13_{-0.44}^{+0.45}$ & $0.86 \pm 0.16_{-0.20}^{+0.19}$ \\
\hline 2 & $33.31 \pm 0.43_{-0.57}^{+0.65}$ & $27.99 \pm 0.24_{-0.21}^{+0.21}$ & $17.14 \pm 0.30_{-0.51}^{+0.44}$ & $10.07 \pm 0.09_{-0.38}^{+0.38}$ & $5.50 \pm 0.13_{-0.38}^{+0.39}$ & $3.38 \pm 0.18_{-0.27}^{+0.28}$ & $2.11 \pm 0.25_{-0.35}^{+0.38}$ \\
\hline 3 & $14.50 \pm 0.26_{-0.39}^{+0.39}$ & $26.40 \pm 0.23_{-0.49}^{+0.50}$ & $24.13 \pm 0.36_{-0.37}^{+0.25}$ & $17.08 \pm 0.12_{-0.20}^{+0.19}$ & $10.95 \pm 0.19_{-0.32}^{+0.29}$ & $7.14 \pm 0.27_{-0.42}^{+0.48}$ & $4.45 \pm 0.38_{-0.63}^{+0.65}$ \\
\hline 4 & $3.46 \pm 0.12_{-0.18}^{+0.18}$ & $15.27 \pm 0.18_{-0.42}^{+0.42}$ & $21.62 \pm 0.34_{-0.66}^{+0.65}$ & $19.82 \pm 0.12_{-0.27}^{+0.18}$ & $14.65 \pm 0.22_{-0.21}^{+0.11}$ & $11.05 \pm 0.34_{-0.25}^{+0.30}$ & $7.36 \pm 0.48_{-0.60}^{+0.63}$ \\
\hline 5 & $0.54 \pm 0.04_{-0.03}^{+0.04}$ & $6.14 \pm 0.11_{-0.40}^{+0.42}$ & $14.72 \pm 0.29_{-0.71}^{+0.73}$ & $18.41 \pm 0.12_{-0.45}^{+0.43}$ & $16.90 \pm 0.23_{-0.15}^{+0.16}$ & $14.03 \pm 0.38_{-0.25}^{+0.26}$ & $10.19 \pm 0.56_{-0.33}^{+0.42}$ \\
\hline 6 & $0.07 \pm 0.02_{-0.01}^{+0.01}$ & $1.88 \pm 0.06_{-0.14}^{+0.14}$ & $7.55 \pm 0.20_{-0.40}^{+0.45}$ & $13.36 \pm 0.10_{-0.48}^{+0.47}$ & $15.42 \pm 0.22_{-0.30}^{+0.30}$ & $14.17 \pm 0.38_{-0.36}^{+0.29}$ & $11.66 \pm 0.60_{-0.27}^{+0.37}$ \\
\hline 7 & & $0.43 \pm 0.02_{-0.03}^{+0.03}$ & $3.16 \pm 0.13_{-0.19}^{+0.22}$ & $8.30 \pm 0.08_{-0.26}^{+0.30}$ & $12.86 \pm 0.20_{-0.41}^{+0.43}$ & $13.22 \pm 0.36_{-0.61}^{+0.60}$ & $11.96 \pm 0.61_{-0.16}^{+0.28}$ \\
\hline 8 & & $0.09 \pm 0.01_{-0.01}^{+0.01}$ & $1.13 \pm 0.08_{-0.08}^{+0.06}$ & $4.36 \pm 0.06_{-0.10}^{+0.17}$ & $8.78 \pm 0.17_{-0.30}^{+0.30}$ & $10.96 \pm 0.33_{-0.44}^{+0.38}$ & $11.26 \pm 0.59_{-0.52}^{+0.66}$ \\
\hline 9 & & & $0.34 \pm 0.04_{-0.02}^{+0.02}$ & $2.09 \pm 0.04_{-0.06}^{+0.10}$ & $5.68 \pm 0.13_{-0.28}^{+0.28}$ & $8.30 \pm 0.28_{-0.41}^{+0.42}$ & $10.10 \pm 0.56_{-0.44}^{+0.47}$ \\
\hline 10 & & & $0.06 \pm 0.01_{-0.01}^{+0.01}$ & $0.85 \pm 0.03_{-0.04}^{+0.06}$ & $3.27 \pm 0.10_{-0.02}^{+0.07}$ & $6.01 \pm 0.25_{-0.08}^{+0.22}$ & $7.66 \pm 0.48_{-0.65}^{+0.57}$ \\
\hline 11 & & & $0.02 \pm 0.01_{-0.00}^{+0.00}$ & $0.33 \pm 0.02_{-0.03}^{+0.04}$ & $1.80 \pm 0.07_{-0.05}^{+0.02}$ & $4.00 \pm 0.20_{-0.07}^{+0.13}$ & $6.32 \pm 0.44_{-0.27}^{+0.18}$ \\
\hline 12 & & & & $0.12 \pm 0.01_{-0.02}^{+0.02}$ & $0.85 \pm 0.05_{-0.09}^{+0.08}$ & $2.53 \pm 0.16_{-0.09}^{+0.10}$ & $4.80 \pm 0.40_{-0.57}^{+0.37}$ \\
\hline 13 & & & & & $0.45 \pm 0.04_{-0.07}^{+0.08}$ & $1.58 \pm 0.12_{-0.07}^{+0.05}$ & $3.46 \pm 0.31_{-0.41}^{+0.35}$ \\
\hline 14 & & & & & $0.19 \pm 0.02_{-0.02}^{+0.02}$ & $0.98 \pm 0.10_{-0.11}^{+0.11}$ & $2.46 \pm 0.26_{-0.13}^{+0.07}$ \\
\hline 15 & & & & & $0.09 \pm 0.02_{-0.02}^{+0.02}$ & $0.48 \pm 0.06_{-0.02}^{+0.05}$ & $1.77 \pm 0.23_{-0.37}^{+0.23}$ \\
\hline 16 & & & & & $0.03 \pm 0.01_{-0.01}^{+0.01}$ & $0.26 \pm 0.05_{-0.03}^{+0.03}$ & $1.17 \pm 0.19_{-0.11}^{+0.09}$ \\
\hline 17 & & & & & $0.02 \pm 0.01_{-0.02}^{+0.01}$ & $0.14 \pm 0.04_{-0.04}^{+0.03}$ & $0.74 \pm 0.15_{-0.14}^{+0.13}$ \\
\hline 18 & & & & & $0.01 \pm 0.01_{-0.00}^{+0.00}$ & $0.06 \pm 0.02_{-0.02}^{+0.02}$ & $0.65 \pm 0.15_{-0.07}^{+0.18}$ \\
\hline 19 & & & & & & & $0.30 \pm 0.08_{-0.11}^{+0.07}$ \\
\hline 20 & & & & & & & $0.20 \pm 0.06_{-0.05}^{+0.17}$ \\
\hline
\end{tabular}

Table 1: Multiplicity distributions $100 \cdot 1 / N d N / d n_{c h}$ measured in the current region of the Breit frame in bins of $2 \cdot E_{\mathrm{B}}^{\mathrm{cr}}$. The first errors are statistical and the second are the systematic uncertainties. 


\begin{tabular}{|c|c|c|c|c|}
\hline$M_{\text {eff }}(\mathrm{GeV})$ & $1.5-4$ & $4-8$ & $8-12$ & $12-20$ \\
\hline$n_{\mathrm{ch}}=0$ & $1.90 \pm 0.05_{-0.34}^{+0.34}$ & $0.23 \pm 0.04_{-0.07}^{+0.07}$ & $0.04 \pm 0.02_{-0.01}^{+0.02}$ & $0.02 \pm 0.02_{-0.01}^{+0.01}$ \\
\hline 1 & $9.81 \pm 0.13_{-0.50}^{+0.47}$ & $1.68 \pm 0.12_{-0.22}^{+0.24}$ & $0.40 \pm 0.05_{-0.02}^{+0.02}$ & $0.13 \pm 0.05_{-0.02}^{+0.02}$ \\
\hline 2 & $21.73 \pm 0.20_{-0.32}^{+0.28}$ & $5.57 \pm 0.24_{-0.26}^{+0.31}$ & $1.26 \pm 0.09_{-0.07}^{+0.04}$ & $0.56 \pm 0.13_{-0.21}^{+0.21}$ \\
\hline 3 & $28.19 \pm 0.23_{-0.29}^{+0.28}$ & $12.12 \pm 0.37_{-0.24}^{+0.35}$ & $3.58 \pm 0.16_{-0.14}^{+0.16}$ & $1.49 \pm 0.18_{-0.17}^{+0.19}$ \\
\hline 4 & $21.12 \pm 0.20_{-0.14}^{+0.15}$ & $17.68 \pm 0.47_{-0.40}^{+0.31}$ & $6.73 \pm 0.23_{-0.12}^{+0.09}$ & $3.33 \pm 0.30_{-0.30}^{+0.26}$ \\
\hline 5 & $10.93 \pm 0.14_{-0.25}^{+0.32}$ & $19.78 \pm 0.51_{-0.29}^{+0.21}$ & $11.07 \pm 0.31_{-0.37}^{+0.34}$ & $5.64 \pm 0.39_{-0.18}^{+0.27}$ \\
\hline 6 & $4.40 \pm 0.09_{-0.08}^{+0.13}$ & $17.11 \pm 0.48_{-0.69}^{+0.69}$ & $14.17 \pm 0.37_{-0.20}^{+0.011}$ & $8.38 \pm 0.50_{-0.12}^{+0.24}$ \\
\hline 7 & $1.43 \pm 0.05_{-0.03}^{+0.05}$ & $12.41 \pm 0.41_{-0.18}^{+0.17}$ & $16.01 \pm 0.40_{-0.42}^{+0.39}$ & $10.47 \pm 0.58_{-0.35}^{+0.40}$ \\
\hline 8 & $0.38 \pm 0.02_{-0.01}^{+0.02}$ & $7.27 \pm 0.31_{-0.24}^{+0.25}$ & $14.40 \pm 0.38_{-0.46}^{+0.46}$ & $12.16 \pm 0.66_{-0.42}^{+0.43}$ \\
\hline 9 & $0.09 \pm 0.01_{-0.01}^{+0.01}$ & $3.51 \pm 0.23_{-0.46}^{+0.47}$ & $11.74 \pm 0.35_{-0.26}^{+0.29}$ & $12.75 \pm 0.68_{-0.17}^{+0.27}$ \\
\hline 10 & & $1.63 \pm 0.15_{-0.16}^{+0.17}$ & $8.46 \pm 0.30_{-0.29}^{+0.33}$ & $11.80 \pm 0.69_{-0.26}^{+0.28}$ \\
\hline 11 & & $0.66 \pm 0.09_{-0.08}^{+0.09}$ & $5.50 \pm 0.24_{-0.21}^{+0.25}$ & $10.03 \pm 0.63_{-0.12}^{+0.33}$ \\
\hline 12 & & $0.26 \pm 0.06_{-0.05}^{+0.05}$ & $3.16 \pm 0.18_{-0.31}^{+0.32}$ & $7.74 \pm 0.57_{-0.34}^{+0.25}$ \\
\hline 13 & & $0.04 \pm 0.02_{-0.01}^{+0.01}$ & $1.76 \pm 0.13_{-0.20}^{+0.24}$ & $5.56 \pm 0.46_{-0.48}^{+0.21}$ \\
\hline 14 & & $0.03 \pm 0.02_{-0.01}^{+0.01}$ & $0.94 \pm 0.09_{-0.11}^{+0.13}$ & $4.02 \pm 0.40_{-0.21}^{+0.33}$ \\
\hline 15 & & $0.01 \pm 0.01_{-0.01}^{+0.01}$ & $0.41 \pm 0.06_{-0.12}^{+0.13}$ & $2.50 \pm 0.31_{-0.22}^{+0.11}$ \\
\hline 16 & & & $0.19 \pm 0.04_{-0.04}^{+0.04}$ & $1.44 \pm 0.25_{-0.14}^{+0.07}$ \\
\hline 17 & & & $0.10 \pm 0.03_{-0.05}^{+0.03}$ & $0.89 \pm 0.19_{-0.23}^{+0.21}$ \\
\hline 18 & & & $0.05 \pm 0.02_{-0.02}^{+0.03}$ & $0.52 \pm 0.14_{-0.13}^{+0.13}$ \\
\hline 19 & & & $0.01 \pm 0.01_{-0.00}^{+0.00}$ & $0.22 \pm 0.09_{-0.11}^{+0.11}$ \\
\hline 20 & & & & $0.19 \pm 0.09_{-0.10}^{+0.13}$ \\
\hline
\end{tabular}

Table 2: $\quad$ Multiplicity distributions $100 \cdot 1 / N d N / d n_{c h}$ measured in the current region of the Breit frame in bins of $M_{\mathrm{eff}}$. The first errors are statistical and the second are the systematic uncertainties. 


\begin{tabular}{|c|c|c|c|}
\hline$W(\mathrm{GeV})$ & $70-100$ & $100-150$ & $150-225$ \\
\hline$n_{\mathrm{ch}}=0$ & $0.09 \pm 0.03_{-0.01}^{+0.01}$ & $0.11 \pm 0.03_{-0.02}^{+0.02}$ & $0.09 \pm 0.02_{-0.04}^{+0.05}$ \\
\hline 1 & $0.37 \pm 0.04_{-0.13}^{+0.13}$ & $0.26 \pm 0.03_{-0.10}^{+0.10}$ & $0.25 \pm 0.04_{-0.17}^{+0.17}$ \\
\hline 2 & $1.50 \pm 0.07_{-0.28}^{+0.28}$ & $1.02 \pm 0.06_{-0.23}^{+0.23}$ & $0.81 \pm 0.05_{-0.19}^{+0.19}$ \\
\hline 3 & $2.58 \pm 0.09_{-0.36}^{+0.36}$ & $1.55 \pm 0.06_{-0.17}^{+0.17}$ & $0.99 \pm 0.05_{-0.17}^{+0.18}$ \\
\hline 4 & $5.22 \pm 0.13_{-0.52}^{+0.52}$ & $3.60 \pm 0.09_{-0.69}^{+0.69}$ & $2.43 \pm 0.08_{-0.66}^{+0.66}$ \\
\hline 5 & $6.47 \pm 0.14_{-0.38}^{+0.39}$ & $4.43 \pm 0.10_{-0.13}^{+0.14}$ & $3.00 \pm 0.08_{-0.06}^{+0.07}$ \\
\hline 6 & $8.97 \pm 0.16_{-0.58}^{+0.58}$ & $6.71 \pm 0.12_{-0.85}^{+0.85}$ & $4.72 \pm 0.10_{-0.80}^{+0.80}$ \\
\hline 7 & $9.57 \pm 0.17_{-0.09}^{+0.12}$ & $7.40 \pm 0.13_{-0.18}^{+0.19}$ & $5.51 \pm 0.11_{-0.23}^{+0.24}$ \\
\hline 8 & $9.89 \pm 0.16_{-0.78}^{+0.78}$ & $8.41 \pm 0.13_{-0.89}^{+0.89}$ & $6.82 \pm 0.12_{-0.87}^{+0.87}$ \\
\hline 9 & $9.53 \pm 0.16_{-0.53}^{+0.53}$ & $8.45 \pm 0.13_{-0.51}^{+0.51}$ & $7.18 \pm 0.12_{-0.57}^{+0.57}$ \\
\hline 10 & $8.73 \pm 0.16_{-0.70}^{+0.70}$ & $8.40 \pm 0.13_{-0.85}^{+0.85}$ & $7.61 \pm 0.13_{-0.74}^{+0.74}$ \\
\hline 11 & $7.82 \pm 0.15_{-0.41}^{+0.41}$ & $8.05 \pm 0.13_{-0.41}^{+0.40}$ & $7.54 \pm 0.13_{-0.57}^{+0.57}$ \\
\hline 12 & $6.63 \pm 0.14_{-0.16}^{+0.16}$ & $7.31 \pm 0.12_{-0.49}^{+0.49}$ & $7.41 \pm 0.13_{-0.58}^{+0.58}$ \\
\hline 13 & $5.50 \pm 0.13_{-0.12}^{+0.11}$ & $6.46 \pm 0.12_{-0.15}^{+0.15}$ & $6.82 \pm 0.12_{-0.30}^{+0.30}$ \\
\hline 14 & $4.32 \pm 0.12_{-0.15}^{+0.12}$ & $5.53 \pm 0.11_{-0.02}^{+0.03}$ & $6.33 \pm 0.12_{-0.31}^{+0.31}$ \\
\hline 15 & $3.45 \pm 0.11_{-0.25}^{+0.25}$ & $4.72 \pm 0.10_{-0.19}^{+0.19}$ & $5.69 \pm 0.11_{-0.05}^{+0.05}$ \\
\hline 16 & $2.58 \pm 0.09_{-0.37}^{+0.37}$ & $3.90 \pm 0.10_{-0.27}^{+0.27}$ & $4.93 \pm 0.11_{-0.03}^{+0.00}$ \\
\hline 17 & $1.96 \pm 0.09_{-0.44}^{+0.44}$ & $3.16 \pm 0.09_{-0.44}^{+0.44}$ & $4.22 \pm 0.10_{-0.30}^{+0.30}$ \\
\hline 18 & $1.46 \pm 0.08_{-0.45}^{+0.45}$ & $2.58 \pm 0.08_{-0.45}^{+0.45}$ & $3.48 \pm 0.09_{-0.30}^{+0.30}$ \\
\hline 19 & $1.05 \pm 0.07_{-0.35}^{+0.35}$ & $1.98 \pm 0.08_{-0.51}^{+0.51}$ & $2.95 \pm 0.09_{-0.37}^{+0.37}$ \\
\hline 20 & $0.74 \pm 0.06_{-0.34}^{+0.34}$ & $1.50 \pm 0.07_{-0.51}^{+0.51}$ & $2.32 \pm 0.08_{-0.45}^{+0.45}$ \\
\hline 21 & $0.51 \pm 0.06_{-0.25}^{+0.25}$ & $1.14 \pm 0.06_{-0.48}^{+0.48}$ & $1.91 \pm 0.08_{-0.52}^{+0.52}$ \\
\hline 22 & $0.34 \pm 0.05_{-0.19}^{+0.19}$ & $0.90 \pm 0.06_{-0.38}^{+0.38}$ & $1.51 \pm 0.07_{-0.48}^{+0.48}$ \\
\hline 23 & $0.25 \pm 0.05_{-0.16}^{+0.16}$ & $0.65 \pm 0.05_{-0.36}^{+0.36}$ & $1.22 \pm 0.07_{-0.47}^{+0.47}$ \\
\hline 24 & $0.17 \pm 0.04_{-0.12}^{+0.12}$ & $0.46 \pm 0.05_{-0.27}^{+0.27}$ & $0.94 \pm 0.06_{-0.41}^{+0.41}$ \\
\hline 25 & $0.10 \pm 0.04_{-0.08}^{+0.08}$ & $0.36 \pm 0.05_{-0.23}^{+0.23}$ & $0.74 \pm 0.06_{-0.40}^{+0.40}$ \\
\hline 26 & $0.08 \pm 0.03_{-0.06}^{+0.06}$ & $0.28 \pm 0.04_{-0.20}^{+0.20}$ & $0.58 \pm 0.05_{-0.31}^{+0.31}$ \\
\hline 27 & $0.05 \pm 0.03_{-0.04}^{+0.04}$ & $0.19 \pm 0.04_{-0.15}^{+0.15}$ & $0.45 \pm 0.05_{-0.27}^{+0.27}$ \\
\hline 28 & $0.03 \pm 0.02_{-0.03}^{+0.03}$ & $0.15 \pm 0.04_{-0.12}^{+0.12}$ & $0.36 \pm 0.05_{-0.23}^{+0.23}$ \\
\hline 29 & $0.02 \pm 0.02_{-0.02}^{+0.01}$ & $0.10 \pm 0.04_{-0.08}^{+0.08}$ & $0.29 \pm 0.05_{-0.20}^{+0.20}$ \\
\hline 30 & $0.01 \pm 0.03_{-0.02}^{+0.01}$ & $0.07 \pm 0.02_{-0.05}^{+0.05}$ & $0.22 \pm 0.05_{-0.17}^{+0.17}$ \\
\hline 31 & & $0.05 \pm 0.03_{-0.04}^{+0.04}$ & $0.17 \pm 0.04_{-0.12}^{+0.12}$ \\
\hline 32 & & $0.04 \pm 0.03_{-0.03}^{+0.03}$ & $0.13 \pm 0.05_{-0.11}^{+0.11}$ \\
\hline 33 & & $0.03 \pm 0.03_{-0.03}^{+0.03}$ & $0.10 \pm 0.04_{-0.08}^{+0.08}$ \\
\hline 34 & & $0.01 \pm 0.02_{-0.01}^{+0.01}$ & $0.08 \pm 0.03_{-0.06}^{+0.06}$ \\
\hline 35 & & $0.01 \pm 0.01_{-0.01}^{+0.01}$ & $0.05 \pm 0.03_{-0.07}^{+0.05}$ \\
\hline 36 & & & $0.05 \pm 0.03_{-0.04}^{+0.05}$ \\
\hline 37 & & & $0.03 \pm 0.03_{-0.03}^{+0.03}$ \\
\hline 38 & & & $0.02 \pm 0.02_{-0.02}^{+0.02}$ \\
\hline
\end{tabular}

Table 3: Multiplicity distributions $100 \cdot 1 / N d N / d n_{c h}$ measured in the current region of the HCM in bins of $W$. The first errors are statistical and the second are the systematic uncertainties. 


\begin{tabular}{|c|c|c|c|c|c|}
\hline$M_{\text {eff }}(\mathrm{GeV})$ & $1.5-4$ & $4-8$ & $8-12$ & $12-20$ & $20-30$ \\
\hline$n_{\mathrm{ch}}=0$ & $1.44 \pm 0.09_{-0.58}^{+0.58}$ & $0.12 \pm 0.01_{-0.04}^{+0.04}$ & $0.02 \pm 0.01_{-0.00}^{+0.00}$ & & \\
\hline 1 & $6.59 \pm 0.22_{-0.74}^{+0.74}$ & $1.06 \pm 0.04_{-0.06}^{+0.05}$ & $0.18 \pm 0.02_{-0.03}^{+0.03}$ & $0.05 \pm 0.01_{-0.01}^{+0.01}$ & \\
\hline 2 & $16.69 \pm 0.40_{-1.50}^{+1.54}$ & $3.83 \pm 0.09_{-0.13}^{+0.13}$ & $0.76 \pm 0.04_{-0.04}^{+0.01}$ & $0.17 \pm 0.02_{-0.01}^{+0.01}$ & $0.03 \pm 0.02_{-0.01}^{+0.01}$ \\
\hline 3 & $23.29 \pm 0.49_{-0.21}^{+0.27}$ & $8.91 \pm 0.14_{-0.22}^{+0.27}$ & $2.27 \pm 0.08_{-0.10}^{+0.03}$ & $0.54 \pm 0.04_{-0.03}^{+0.01}$ & $0.13 \pm 0.04_{-0.03}^{+0.05}$ \\
\hline 4 & $24.20 \pm 0.52_{-1.28}^{+1.26}$ & $15.01 \pm 0.19_{-0.82}^{+0.81}$ & $5.15 \pm 0.12_{-0.38}^{+0.32}$ & $1.32 \pm 0.06_{-0.09}^{+0.08}$ & $0.31 \pm 0.06_{-0.06}^{+0.08}$ \\
\hline 5 & $15.49 \pm 0.41_{-0.86}^{+0.87}$ & $18.40 \pm 0.21_{-0.21}^{+0.14}$ & $8.84 \pm 0.16_{-0.48}^{+0.46}$ & $2.81 \pm 0.09_{-0.20}^{+0.19}$ & $0.78 \pm 0.10_{-0.07}^{+0.10}$ \\
\hline 6 & $8.07 \pm 0.29_{-0.32}^{+0.34}$ & $18.13 \pm 0.22_{-0.71}^{+0.71}$ & $12.51 \pm 0.19_{-0.65}^{+0.64}$ & $4.81 \pm 0.12_{-0.43}^{+0.41}$ & $1.49 \pm 0.13_{-0.07}^{+0.13}$ \\
\hline 7 & $3.03 \pm 0.17_{-0.18}^{+0.16}$ & $14.32 \pm 0.19_{-0.34}^{+0.32}$ & $14.61 \pm 0.21_{-0.79}^{+0.79}$ & $7.30 \pm 0.15_{-0.69}^{+0.68}$ & $2.43 \pm 0.18_{-0.23}^{+0.18}$ \\
\hline 8 & $0.95 \pm 0.09_{-0.12}^{+0.13}$ & $9.62 \pm 0.16_{-0.24}^{+0.26}$ & $14.62 \pm 0.21_{-0.59}^{+0.60}$ & $9.38 \pm 0.18_{-0.69}^{+0.68}$ & $3.91 \pm 0.22_{-0.59}^{+0.65}$ \\
\hline 9 & $0.20 \pm 0.04_{-0.07}^{+0.06}$ & $5.54 \pm 0.12_{-0.49}^{+0.50}$ & $12.97 \pm 0.20_{-0.16}^{+0.20}$ & $11.03 \pm 0.19_{-0.86}^{+0.85}$ & $5.56 \pm 0.28_{-0.93}^{+0.95}$ \\
\hline 10 & $0.05 \pm 0.02_{-0.01}^{+0.03}$ & $2.82 \pm 0.09_{-0.33}^{+0.34}$ & $10.32 \pm 0.19_{-0.27}^{+0.28}$ & $11.53 \pm 0.20_{-0.63}^{+0.63}$ & $6.97 \pm 0.31_{-1.13}^{+1.10}$ \\
\hline 11 & & $1.36 \pm 0.06_{-0.28}^{+0.28}$ & $7.24 \pm 0.16_{-0.44}^{+0.46}$ & $11.18 \pm 0.20_{-0.59}^{+0.59}$ & $8.01 \pm 0.33_{-1.25}^{+1.23}$ \\
\hline 12 & & $0.57 \pm 0.04_{-0.13}^{+0.13}$ & $4.74 \pm 0.13_{-0.70}^{+0.71}$ & $10.07 \pm 0.20_{-0.21}^{+0.20}$ & $8.48 \pm 0.35_{-1.04}^{+1.05}$ \\
\hline 13 & & $0.19 \pm 0.03_{-0.07}^{+0.07}$ & $2.73 \pm 0.10_{-0.58}^{+0.59}$ & $8.44 \pm 0.18_{-0.24}^{+0.27}$ & $9.26 \pm 0.36_{-1.36}^{+1.36}$ \\
\hline 14 & & $0.08 \pm 0.02_{-0.02}^{+0.02}$ & $1.51 \pm 0.08_{-0.37}^{+0.38}$ & $6.58 \pm 0.16_{-0.51}^{+0.52}$ & $8.90 \pm 0.38_{-0.71}^{+0.72}$ \\
\hline 15 & & $0.03 \pm 0.01_{-0.01}^{+0.00}$ & $0.83 \pm 0.06_{-0.28}^{+0.28}$ & $4.92 \pm 0.15_{-0.59}^{+0.60}$ & $8.09 \pm 0.36_{-0.35}^{+0.37}$ \\
\hline 16 & & & $0.35 \pm 0.04_{-0.14}^{+0.14}$ & $3.53 \pm 0.13_{-0.65}^{+0.66}$ & $7.57 \pm 0.36_{-0.21}^{+0.21}$ \\
\hline 17 & & & $0.20 \pm 0.03_{-0.08}^{+0.08}$ & $2.43 \pm 0.11_{-0.63}^{+0.64}$ & $6.41 \pm 0.35_{-0.45}^{+0.47}$ \\
\hline 18 & & & $0.08 \pm 0.02_{-0.03}^{+0.03}$ & $1.56 \pm 0.10_{-0.56}^{+0.56}$ & $5.17 \pm 0.33_{-0.90}^{+0.86}$ \\
\hline 19 & & & $0.03 \pm 0.02_{-0.02}^{+0.02}$ & $0.96 \pm 0.08_{-0.36}^{+0.36}$ & $4.08 \pm 0.29_{-0.71}^{+0.65}$ \\
\hline 20 & & & $0.01 \pm 0.01_{-0.01}^{+0.01}$ & $0.59 \pm 0.06_{-0.25}^{+0.25}$ & $3.47 \pm 0.29_{-0.95}^{+0.96}$ \\
\hline 21 & & & & $0.36 \pm 0.05_{-0.18}^{+0.18}$ & $2.63 \pm 0.27_{-0.98}^{+0.99}$ \\
\hline 22 & & & & $0.18 \pm 0.04_{-0.11}^{+0.12}$ & $1.83 \pm 0.24_{-0.72}^{+0.72}$ \\
\hline 23 & & & & $0.12 \pm 0.04_{-0.08}^{+0.09}$ & $1.39 \pm 0.23_{-0.75}^{+0.77}$ \\
\hline 24 & & & & $0.07 \pm 0.02_{-0.04}^{+0.04}$ & $0.96 \pm 0.20_{-0.63}^{+0.62}$ \\
\hline 25 & & & & $0.04 \pm 0.02_{-0.02}^{+0.02}$ & $0.66 \pm 0.20_{-0.52}^{+0.50}$ \\
\hline 26 & & & & $0.02 \pm 0.02_{-0.02}^{+0.01}$ & $0.53 \pm 0.16_{-0.33}^{+0.33}$ \\
\hline 27 & & & & & $0.34 \pm 0.16_{-0.22}^{+0.27}$ \\
\hline 28 & & & & & $0.28 \pm 0.20_{-0.26}^{+0.26}$ \\
\hline 29 & & & & & $0.13 \pm 0.11_{-0.13}^{+0.11}$ \\
\hline 30 & & & & & $0.09 \pm 0.07_{-0.12}^{+0.07}$ \\
\hline
\end{tabular}

Table 4: Multiplicity distributions $100 \cdot 1 / N d N / d n_{c h}$ measured in the current region of the HCM frame in bins of $M_{\mathrm{eff}}$. The first errors are statistical and the second are the systematic uncertainties. 


\begin{tabular}{|c|c|c|}
\hline $2 \cdot E_{\mathrm{B}}^{\mathrm{cr}}(\mathrm{GeV})$ & $\left\langle n_{\mathrm{ch}}\right\rangle\left(K^{0}, \Lambda\right.$ stable $)$ & $\left\langle n_{\mathrm{ch}}\right\rangle\left(K^{0}, \Lambda\right.$ decay $)$ \\
\hline \hline 2.9 & $1.50 \pm 0.01_{-0.00}^{+0.04}$ & $1.63 \pm 0.01_{-0.01}^{+0.05}$ \\
5.9 & $2.39 \pm 0.01_{-0.05}^{+0.09}$ & $2.60 \pm 0.01_{-0.05}^{+0.11}$ \\
9.6 & $3.27 \pm 0.01_{-0.08}^{+0.12}$ & $3.55 \pm 0.01_{-0.08}^{+0.15}$ \\
14.8 & $4.17 \pm 0.01_{-0.05}^{+0.10}$ & $4.53 \pm 0.01_{-0.05}^{+0.12}$ \\
23.8 & $5.22 \pm 0.01_{-0.05}^{+0.06}$ & $5.67 \pm 0.01_{-0.05}^{+0.08}$ \\
35.6 & $6.19 \pm 0.03_{-0.07}^{+0.08}$ & $6.68 \pm 0.03_{-0.08}^{+0.08}$ \\
58.1 & $7.46 \pm 0.06_{-0.16}^{+0.14}$ & $8.04 \pm 0.06_{-0.18}^{+0.16}$ \\
\hline \hline$W(\mathrm{GeV})$ & $\left\langle n_{\mathrm{ch}}\right\rangle\left(K^{0}, \Lambda\right.$ stable $)$ & $\left\langle n_{\mathrm{ch}}\right\rangle\left(K^{0}, \Lambda\right.$ decay $)$ \\
\hline \hline 84.6 & $8.72 \pm 0.02_{-0.22}^{+0.22}$ & $9.58 \pm 0.02_{-0.33}^{+0.33}$ \\
123.8 & $10.04 \pm 0.02_{-0.44}^{+0.44}$ & $11.07 \pm 0.02_{-0.61}^{+0.61}$ \\
184.5 & $11.40 \pm 0.03_{-0.58}^{+0.58}$ & $12.59 \pm 0.03_{-0.79}^{+0.79}$ \\
\hline
\end{tabular}

Table 5: Mean charged multiplicity, $\left\langle n_{\mathrm{ch}}\right\rangle$, measured in the current region of the Breit frame as a function of $2 \cdot E_{\mathrm{B}}^{\mathrm{cr}}$ and in the current fragmentation region of the $H C M$ frame as a function of $W$. The first errors are statistical and the second are the systematic uncertainties.

\begin{tabular}{|c|c|c|}
\hline \multicolumn{3}{|c|}{ Current region of the Breit frame } \\
\hline$M_{\text {eff }}(\mathrm{GeV})$ & $\left\langle n_{\mathrm{ch}}\right\rangle\left(K^{0}, \Lambda\right.$ stable $)$ & $\left\langle n_{\mathrm{ch}}\right\rangle\left(K^{0}, \Lambda\right.$ decay $)$ \\
\hline \hline 2.4 & $2.91 \pm 0.01_{-0.03}^{+0.04}$ & $3.17 \pm 0.01_{-0.03}^{+0.04}$ \\
\hline 5.2 & $4.82 \pm 0.02_{-0.03}^{+0.03}$ & $5.26 \pm 0.02_{-0.03}^{+0.03}$ \\
\hline 9.4 & $6.85 \pm 0.02_{-0.05}^{+0.07}$ & $7.45 \pm 0.02_{-0.06}^{+0.08}$ \\
\hline 14.4 & $8.60 \pm 0.06_{-0.09}^{+0.07}$ & $9.29 \pm 0.06_{-0.09}^{+0.14}$ \\
\hline \hline \multicolumn{3}{|c|}{ Current region of the HCM frame } \\
\hline$M_{\text {eff }}(\mathrm{GeV})$ & $\left\langle n_{\mathrm{ch}}\right\rangle\left(K^{0}, \Lambda\right.$ stable $)$ & $\left\langle n_{\mathrm{ch}}\right\rangle\left(K^{0}, \Lambda\right.$ decay $)$ \\
\hline \hline 3.1 & $3.38 \pm 0.02_{-0.02}^{+0.03}$ & $3.64 \pm 0.02_{-0.02}^{+0.05}$ \\
\hline 5.9 & $5.33 \pm 0.01_{-0.07}^{+0.07}$ & $5.77 \pm 0.01_{-0.09}^{+0.09}$ \\
\hline 9.8 & $7.37 \pm 0.02_{-0.12}^{+0.13}$ & $8.05 \pm 0.01_{-0.19}^{+0.19}$ \\
\hline 15.1 & $9.86 \pm 0.02_{-0.23}^{+0.23}$ & $10.84 \pm 0.02_{-0.36}^{+0.36}$ \\
\hline 23.5 & $12.83 \pm 0.06_{-0.54}^{+0.53}$ & $14.17 \pm 0.05_{-0.80}^{+0.80}$ \\
\hline
\end{tabular}

Table 6: $\quad$ Mean charged multiplicity, $\left\langle n_{\mathrm{ch}}\right\rangle$, measured in the current region of the Breit frame and in the current fragmentation region of the HCM frame as a function of $M_{\mathrm{eff}}$. The first errors are statistical and the second are the systematic uncertainties. 
ZEUS

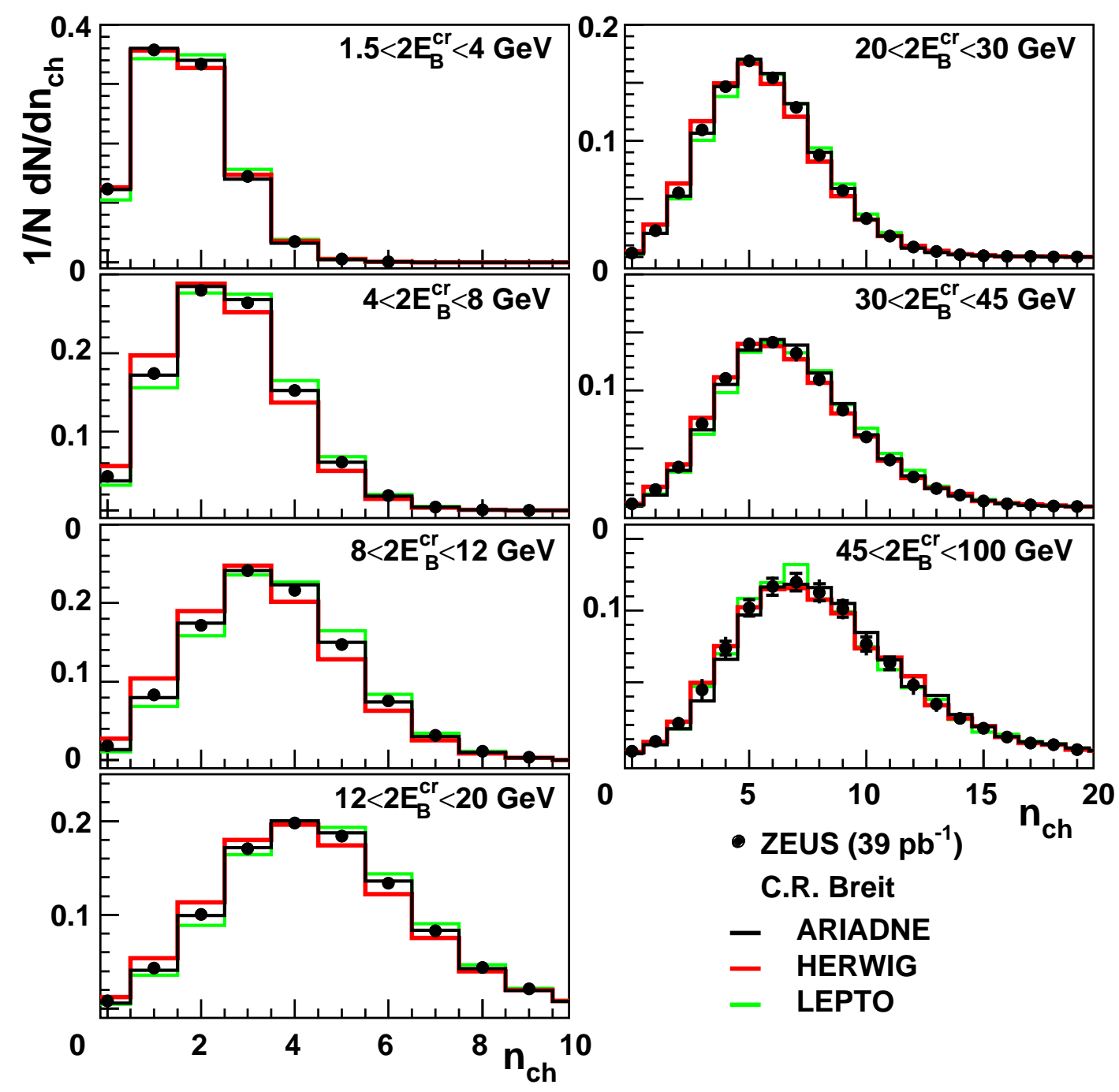

Figure 1: Multiplicity distributions measured in the current region of the Breit frame in bins of $2 \cdot E_{\mathrm{B}}^{\mathrm{cr}}$ (solid circles). The inner error bars represent the statistical uncertainties and the outer error bars the statistical and systematic uncertainties added in quadrature. The predictions (solid lines) of different MC models are also shown. 


\section{ZEUS}

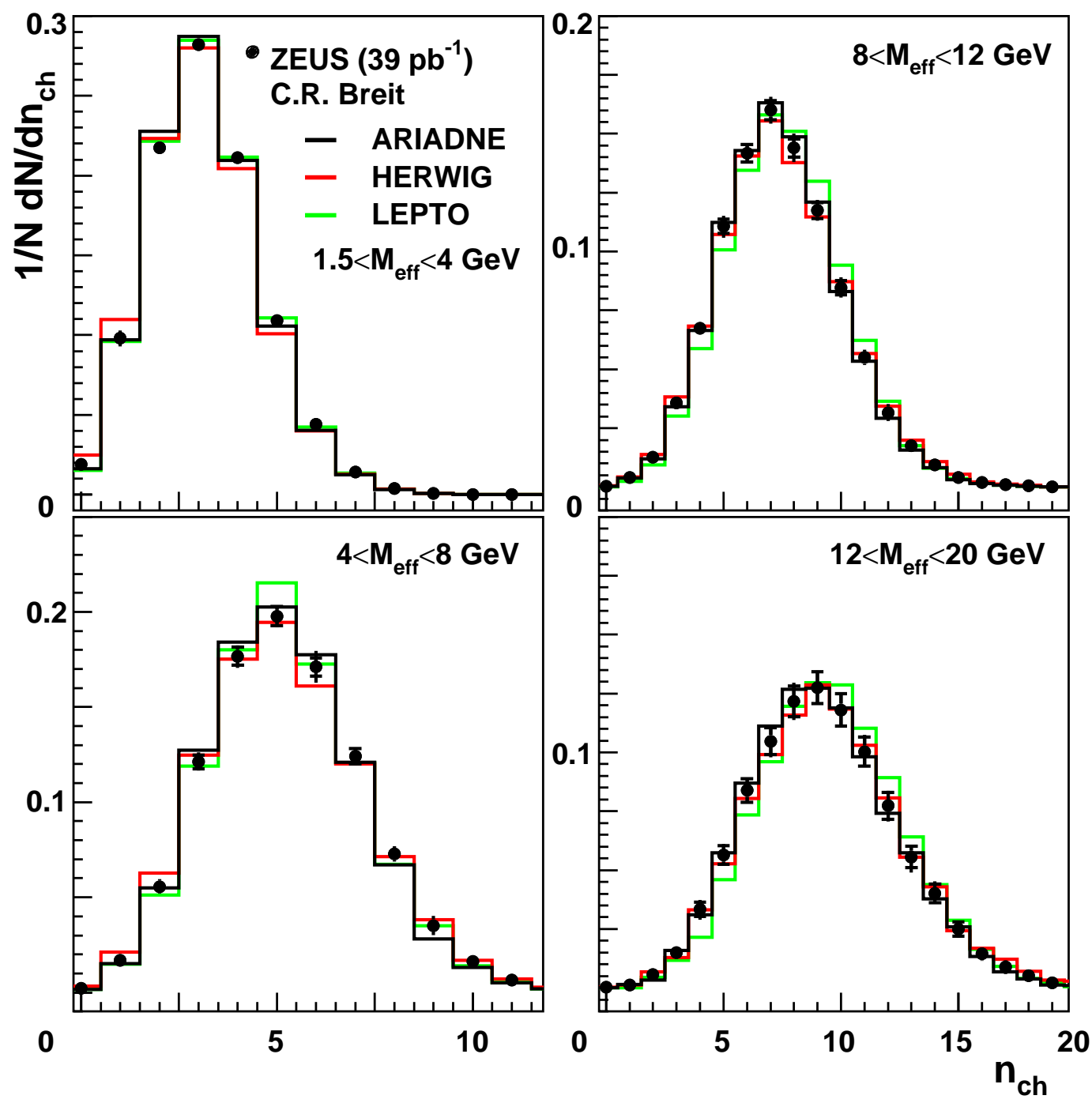

Figure 2: Multiplicity distributions measured in the current region of the Breit frame in bins of $M_{\mathrm{eff}}$ (solid circles). The inner error bars represent the statistical uncertainties and the outer error bars the statistical and systematic uncertainties added in quadrature. The predictions (solid lines) of different MC models are also shown. 


\section{ZEUS}

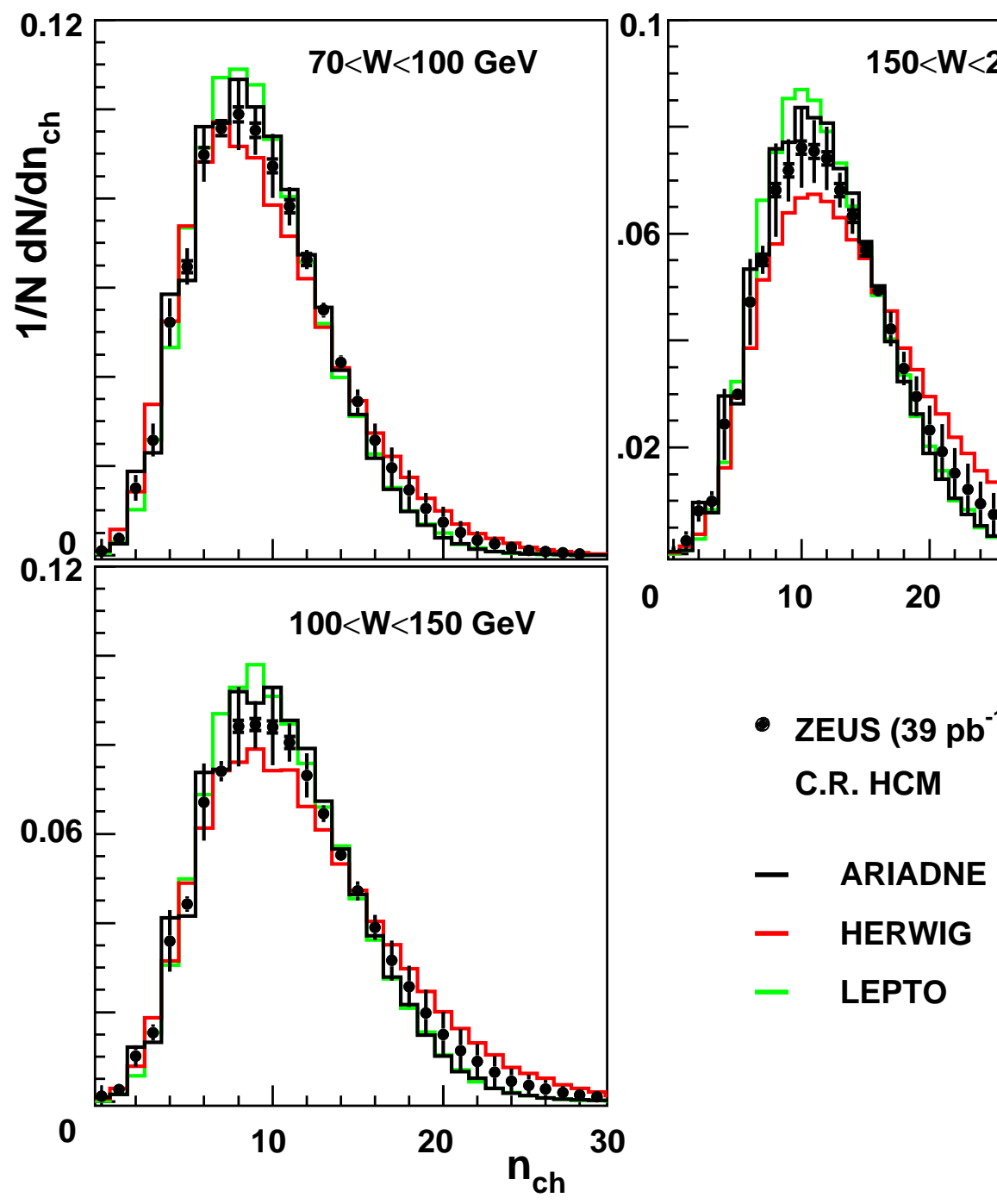

Figure 3: Multiplicity distributions measured in the current region of the HCM frame in bins of $W$ (solid circles). The inner error bars represent the statistical uncertainties and the outer error bars the statistical and systematic uncertainties added in quadrature. The predictions (solid lines) of different $M C$ models are also shown. 


\section{ZEUS}

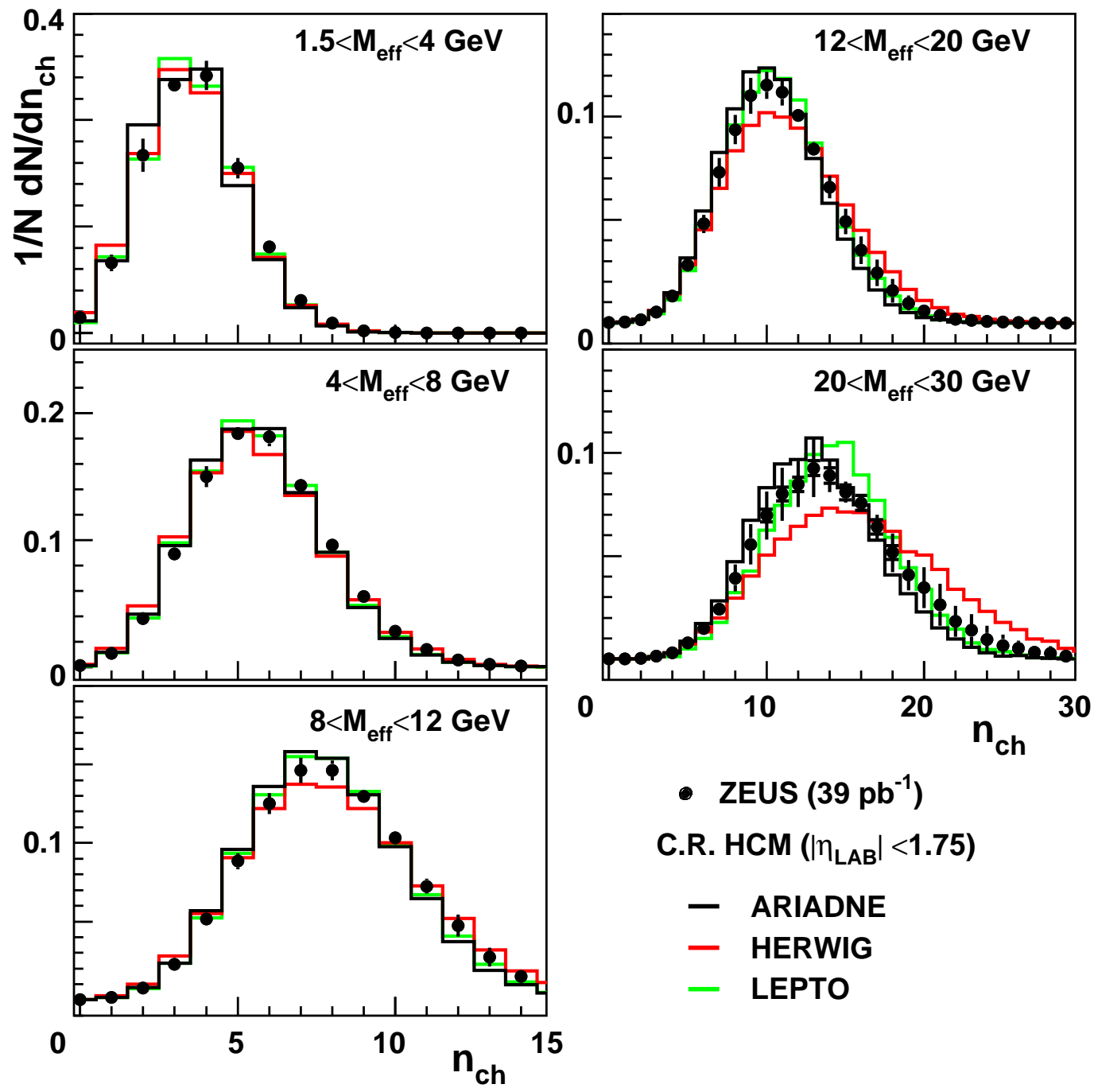

Figure 4: Multiplicity distributions measured in the current region of the HCM frame in bins of $M_{\mathrm{eff}}$ (solid circles). The inner error bars represent the statistical uncertainties and the outer error bars the statistical and systematic uncertainties added in quadrature. The predictions (solid lines) of different MC models are also shown. 


\section{ZEUS}

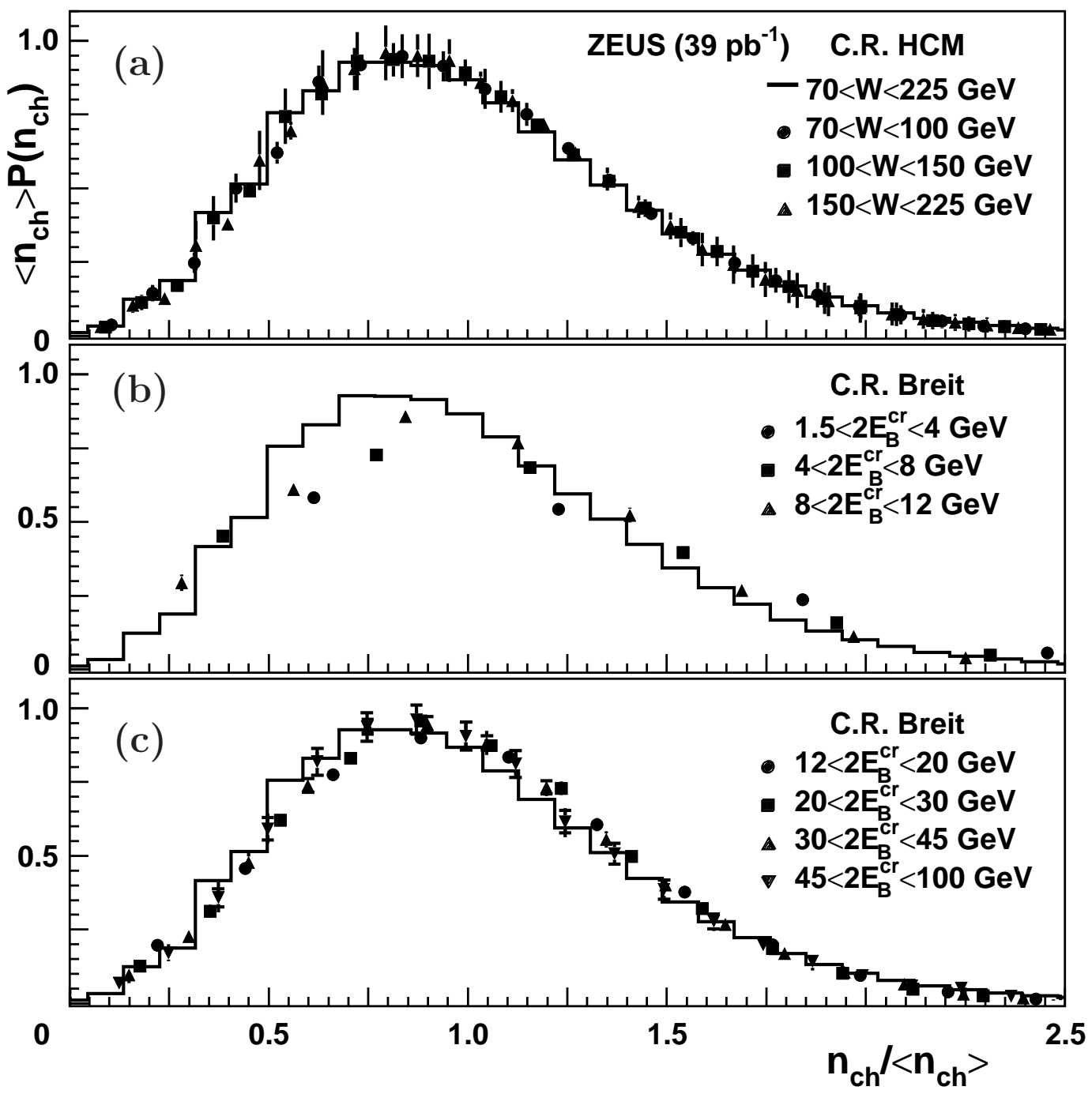

Figure 5: The multiplicity distributions plotted in KNO form. The reference KNO histogram represents the measured distribution in the HCM frame in the entire $W$ range. (a) Multiplicity distributions measured in the current region of the HCM frame in bins of $W$. Multiplicity distributions measured in the current region of the Breit frame in bins of $2 \cdot E_{\mathrm{B}}^{\mathrm{cr}}$ for (b) $1.5<2 \cdot E_{\mathrm{B}}^{\mathrm{cr}}<12 \mathrm{GeV}$ and (c) $12<2 \cdot E_{\mathrm{B}}^{\mathrm{cr}}<$ $100 \mathrm{GeV}$. 


\section{ZEUS}

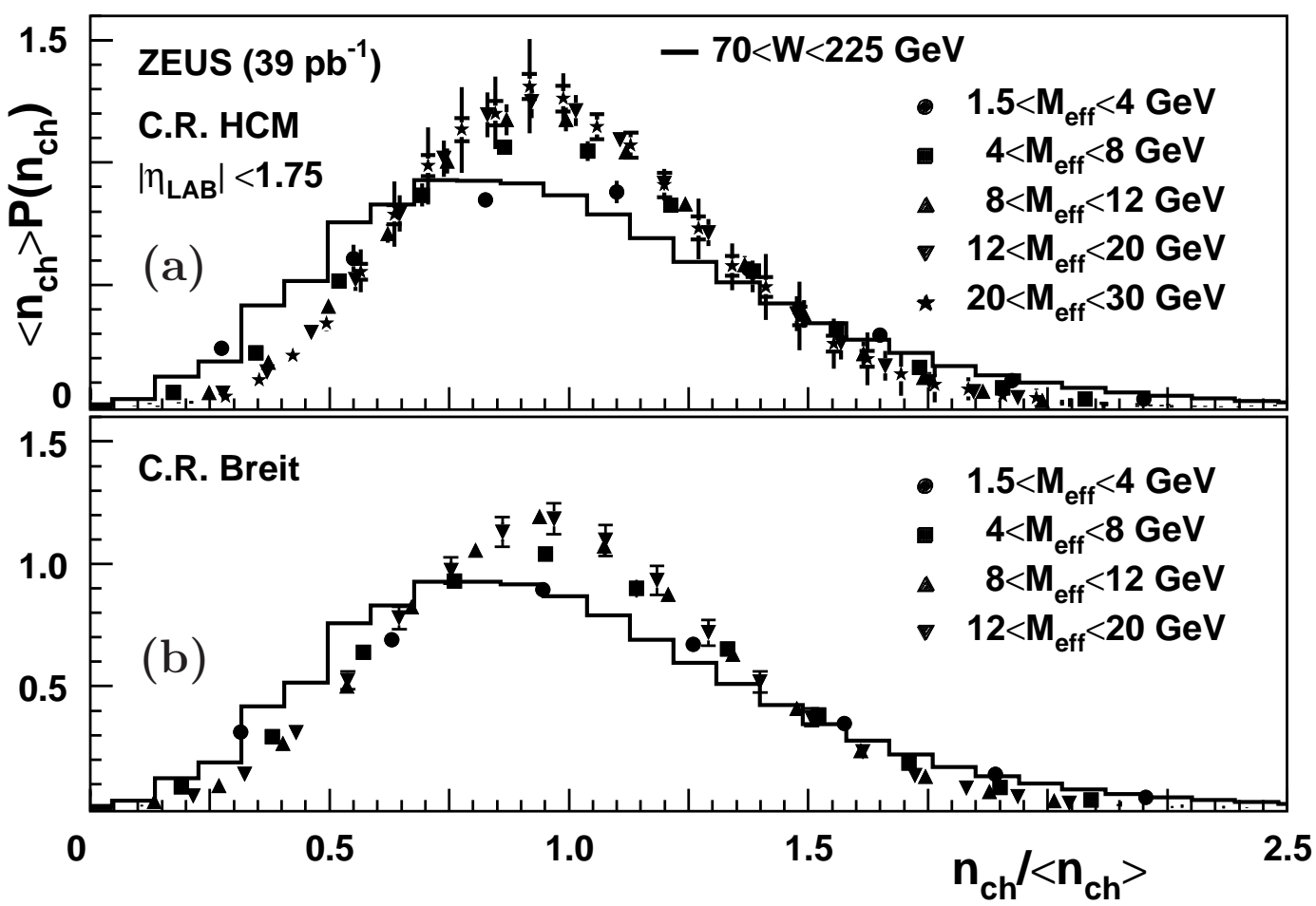

Figure 6: The multiplicity distributions plotted in KNO form and compared to the reference KNO distribution (histogram). The multiplicity distributions are measured in bins of $M_{\mathrm{eff}}$ in the current regions of (a) the HCM frame, restricted in $\eta_{\mathrm{LAB}}$, and (b) the Breit frame. 


\section{ZEUS}

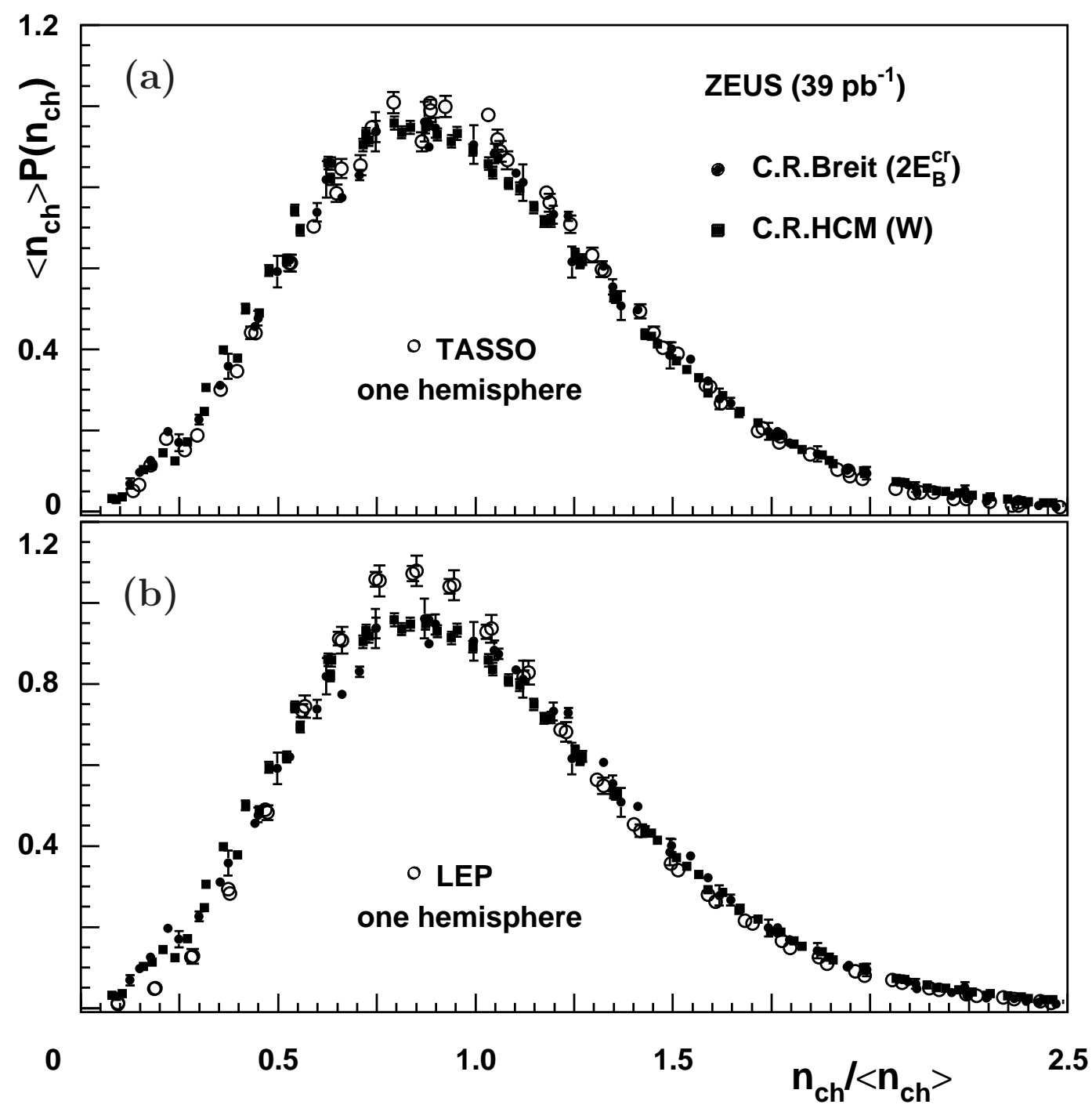

Figure 7: The multiplicity distributions plotted in KNO form and compared to the results of $e^{+} e^{-}$experiments. The solid circles represent the ZEUS data measured in the current region of the Breit frame in bins of $2 \cdot E_{\mathrm{B}}^{\mathrm{cr}}$, for $2 \cdot E_{\mathrm{B}}^{\mathrm{cr}} \geq 12 \mathrm{GeV}$, and the solid squares represent the data measured in the current region of the HCM frame in bins of $W$. Multiplicities measured in one hemisphere of the $e^{+} e^{-}$collision are shown in bins of $\sqrt{s_{e e}}$ for (a) the TASSO experiment [31], in the energy range $14 \leq \sqrt{s_{e e}} \leq 44 \mathrm{GeV}$, and (b) for the LEP experiments [32,33], measured at energy $\sqrt{s_{e e}}=91.2 \mathrm{GeV}$. 


\section{ZEUS}

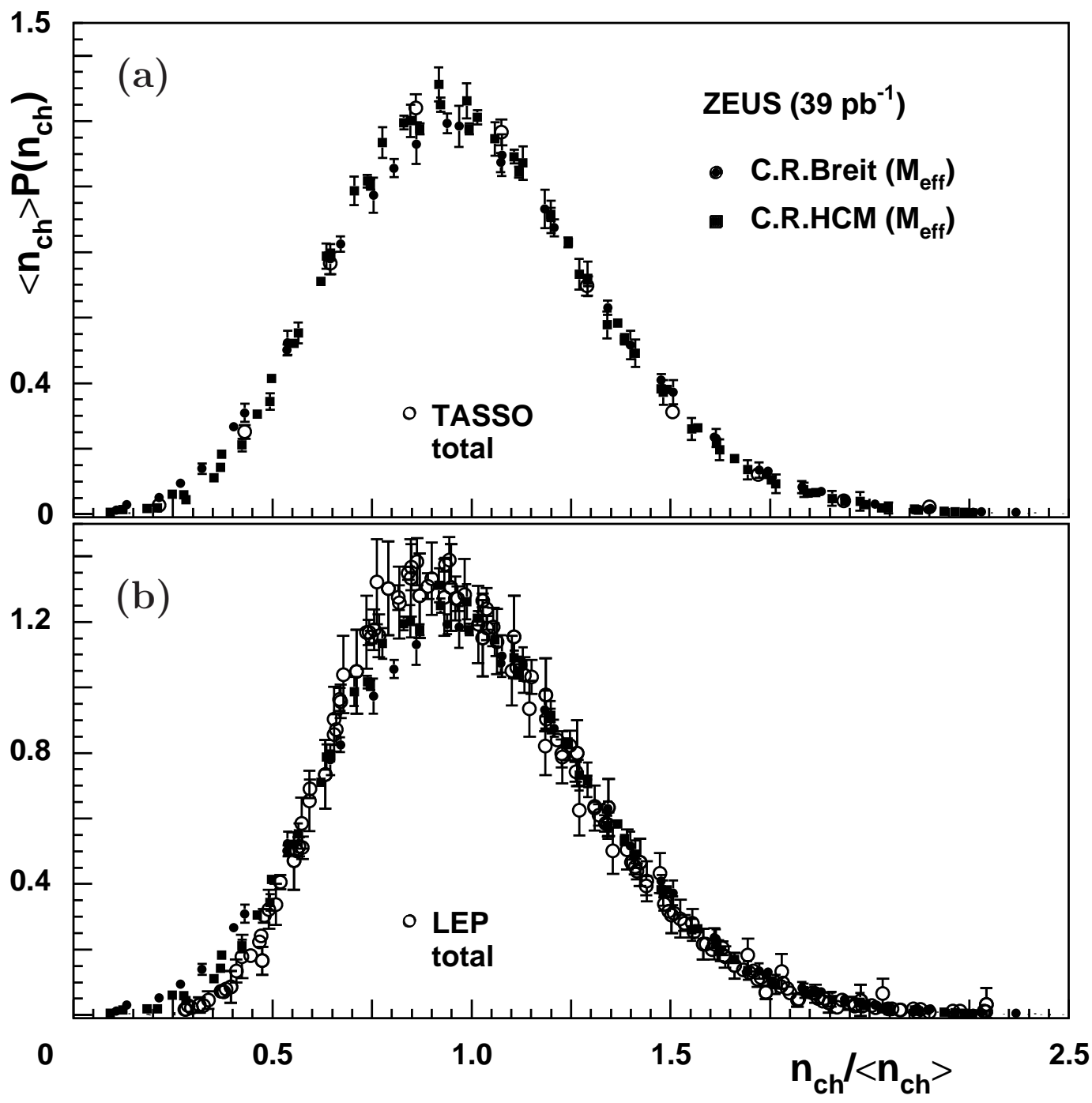

Figure 8: The multiplicity distributions plotted in KNO form and compared to the results of the $e^{+} e^{-}$experiments. The solid circles represent the ZEUS data measured in the current region of the Breit frame and the solid squares represent the data measured in the current region of the HCM frame both in bins of $M_{\mathrm{eff}}$, for $M_{\mathrm{eff}} \geq 8 \mathrm{GeV}$. Charged multiplicities measured for the whole event in $e^{+} e^{-}$ collisions are shown in bins of $\sqrt{s_{e e}}$ for (a) the TASSO experiment [31], in the energy range $14 \leq \sqrt{s_{e e}} \leq 44 \mathrm{GeV}$, and (b) for the LEP experiments [32-34] in the energy range $91.2 \leq \sqrt{s_{e e}} \leq 209 \mathrm{GeV}$. 


\section{ZEUS}

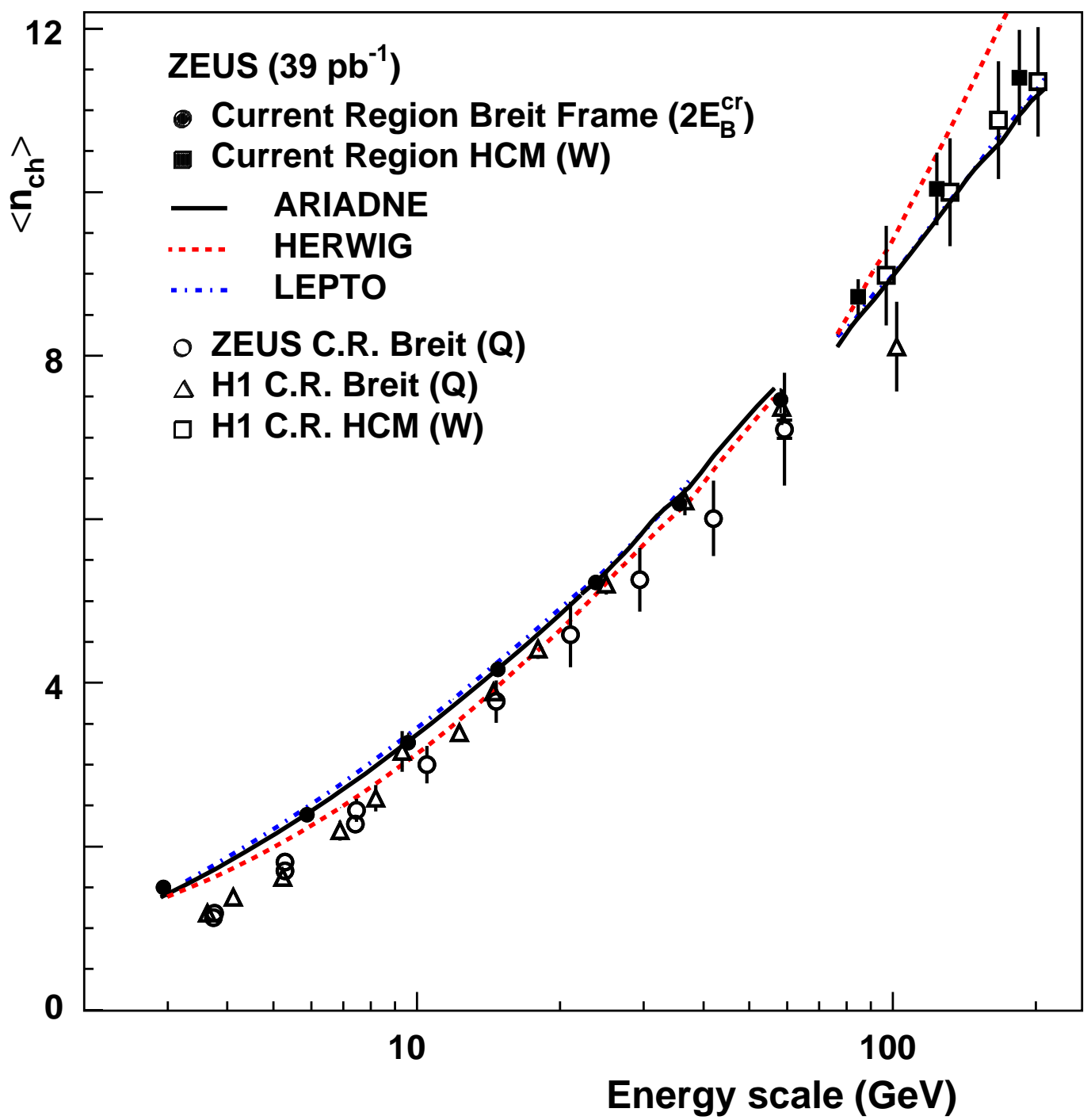

Figure 9: Mean charged multiplicity, $\left\langle n_{\mathrm{ch}}\right\rangle$, in the current region of the Breit frame as a function of $2 \cdot E_{\mathrm{B}}^{\mathrm{cr}}$ and in the current fragmentation region of the $H C M$ frame as a function of $W$. The inner error bars represent the statistical uncertainties, typically smaller than the size of the symbols. The outer error bars represent the quadratic sum of statistical and systematic uncertainties. Also shown are the results of previous HERA measurements [1,2, 4,5] and predictions from ARIADNE, LEPTO and HeRwig. The decay products of $K_{S}^{0}$ and $\Lambda$ are not included. 


\section{ZEUS}

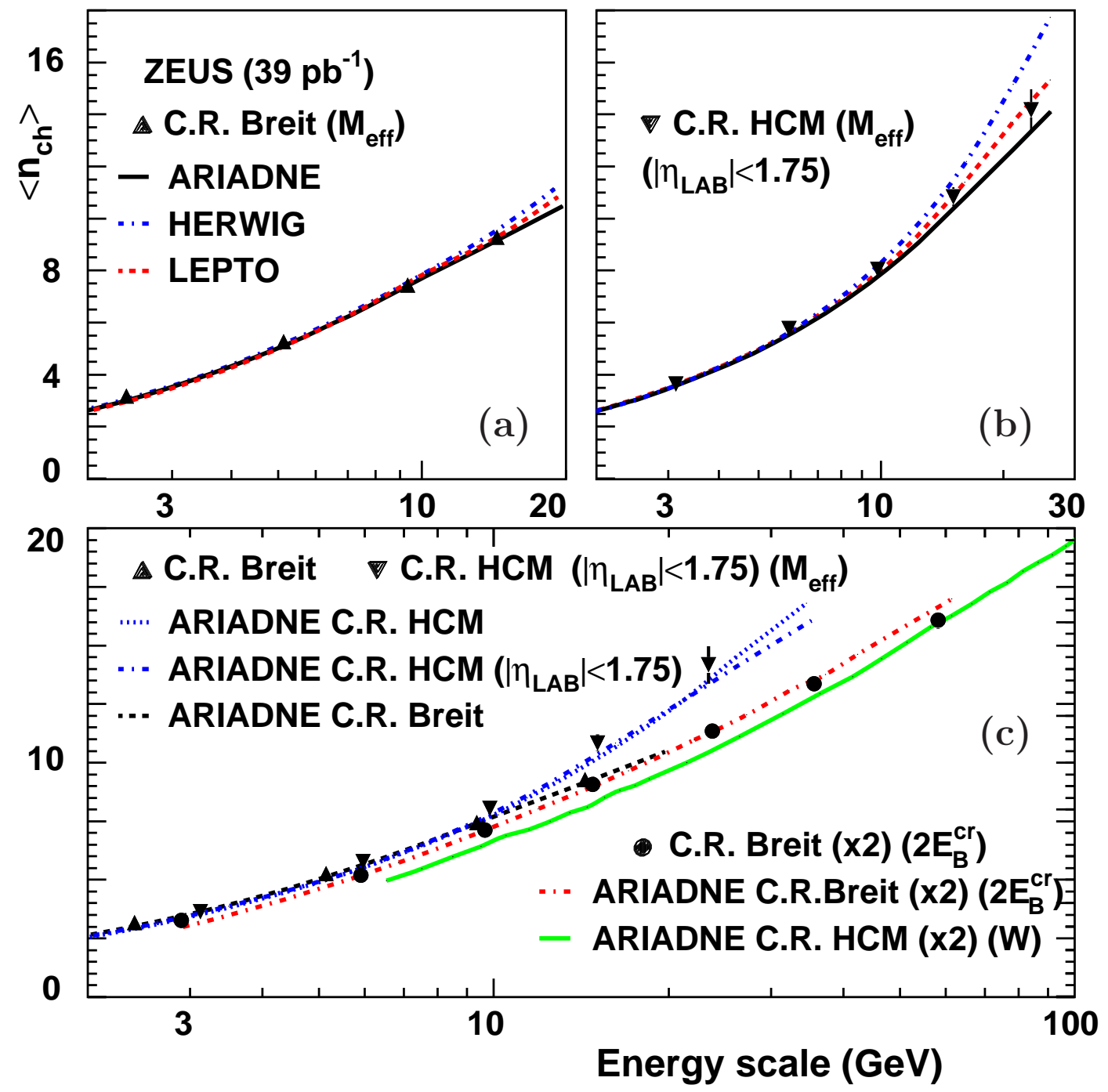

Figure 10: Mean charged multiplicity, $\left\langle n_{\mathrm{ch}}\right\rangle$, measured as a function of $M_{\mathrm{eff}}$ (a) in the current region of the Breit frame and (b) in the current region of the HCM frame compared to MC predictions. (c) Comparison between measurements in the current regions of the Breit and HCM frame as functions of $M_{\mathrm{eff}}$ and with the measurement as a function of $2 \cdot E_{\mathrm{B}}^{\mathrm{cr}}$. The predictions from ARIADNE are also shown. 
ZEUS

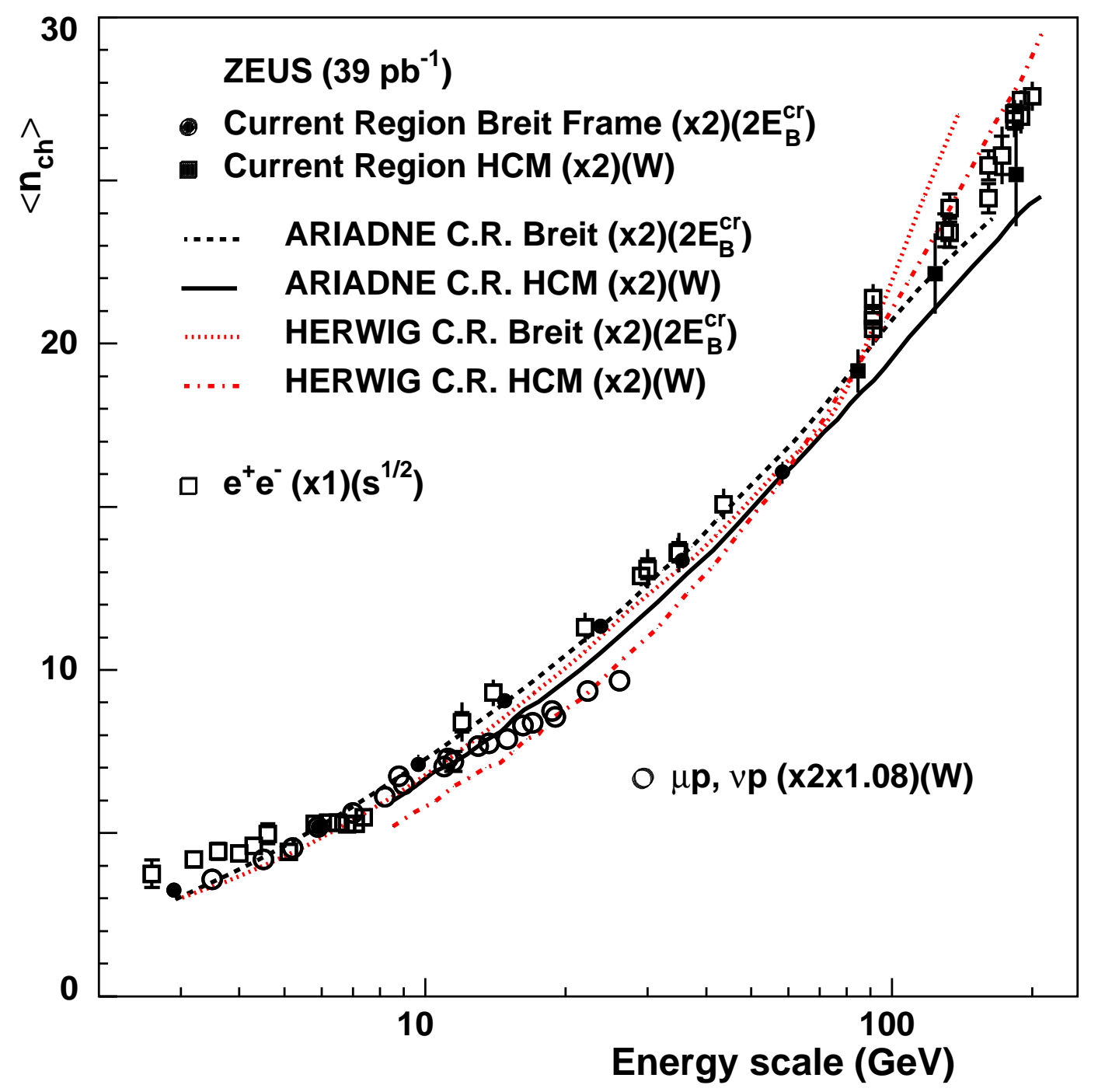

Figure 11: Mean charged multiplicity, $\left\langle n_{\mathrm{ch}}\right\rangle$, in the current region of the Breit frame multiplied by 2 as a function of $2 \cdot E_{\mathrm{B}}^{\mathrm{cr}}$ and in the current region of the $H C M$ frame multiplied by 2 as a function of $W$. The results of $e^{+} e^{-}$[31-35] and fixedtarget DIS experiments [8-10] are shown. The factor 1.08 was estimated using $M C$ predictions to correct the fixed-target data for the decay products of $K_{S}^{0}$ and $\Lambda$. The predictions of ARIADNE and HERWIG are also shown. 